Dirk Peters/Wolfgang Wagner

\title{
Parlamentsvorbehalt oder Exekutivprivileg?
}

\author{
Ursachen unterschiedlicher Entscheidungsverfahren beim Einsatz von \\ Streitkräften
}

Dieser Beitrag geht der Frage nach, warum bei Militäreinsätzen in einigen Demokratien Parlamente ein Vetorecht besitzen, während die Entscheidung in anderen Demokratien allein der Regierung vorbehalten bleibt. Mit Hilfe eines neuen Datensatzes zu 49 Demokratien im Zeitraum 1989 bis 2004 wird gezeigt, dass diese Varianz vor allem auf unterschiedliche Grade äußerer Bedrohung und vergangener Kriegserfahrungen zurückzuführen ist. Außerdem finden sich in aller Regel keine parlamentarischen Vetorechte in Demokratien, die in der britischen Verfassungstradition stehen. Besonderheiten des Demokratisierungsprozesses und die Unterscheidung von parlamentarischen und präsidentiellen Systemen zeigen hingegen keinen nachweisbaren Einfluss.

\section{Einleitung ${ }^{1}$}

Die Debatte um den so genannten Demokratischen Frieden ${ }^{2}$ hat die Aufmerksamkeit der Konfliktforschung (wieder) auf die Bedeutung innerstaatlicher Entscheidungsprozesse für die Sicherheitspolitik von Staaten gelenkt. Die Debatte konzentrierte sich zunächst ganz auf die Erklärung des Doppelbefundes, wonach Demokratien zwar untereinander (fast) keine Kriege führen, aber insgesamt nicht weniger häufig in Kriege verwickelt sind als andere Regime. Die Betrachtung der Kriegshäufigkeit hat allerdings den Blick auf eine Reihe von Verhaltensauffälligkeiten von Demokratien verstellt, die sich auf der monadischen Ebene befinden, sich also generell in der Außen- und Sicherheitspolitik von Demokratien zeigen, ohne dass der Regimetyp ihres Gegenübers einen Einfluss hätte. $\mathrm{Zu}$ den prominentesten Verhaltensauffälligkeiten zählen die Tendenz, Kriege weniger verlustreich zu führen (Rummel 1995; Siverson 1995), das heißt sowohl Opfer unter den eigenen Truppen (Schörnig 2007) als auch bei der gegnerischen Zivilbevölkerung (Watts 2008) zu minimieren, und Kriege überproportional häufig zu gewinnen (Reiter/Stam 2002). Auffällig sind darüber hinaus Unterschiede in den Kriegszielen, die bei Demokratien typischerweise mit der Verteidigung liberaler Werte wie Menschenrechten und Demokratie gerechtfertigt

1 Dieser Beitrag ist im Rahmen des von der Deutschen Stiftung Friedensforschung (DSF) geförderten Projekts »Parlamentarische Kontrolle von Militäreinsätzen« entstanden. Wir danken der DSF für die finanzielle Unterstützung. Für wertvolle inhaltliche Hinweise zum Text danken wir Sandra Dieterich, Hartwig Hummel, Ronald Krebs, Stefan Marschall, Bruce Russett, Harald Schoen und drei anonymen Gutachtern.

2 Die Literatur zum Demokratischen Frieden ist kaum noch überschaubar. Für Überblicksdarstellungen vgl. Russett/Oneal (2001) und Geis/Wagner (2010). 
werden (Doyle 1983; Owen 1994; Hasenclever 2002). Von Autokratien unterscheiden sich Demokratien schließlich durch eine stärker ausgeprägte Bereitschaft und Fähigkeit zu institutionalisierter Zusammenarbeit (vgl. Gaubatz 1996; Martin 2000; Lipson 2003). Die Auswirkungen demokratischer Kontrolle sind also keineswegs auf die dyadische Ebene begrenzt, sondern lassen sich auch auf monadischer Ebene, also in der Außen- und Sicherheitspolitik, nachweisen.

Versuche, solche Verhaltensauffälligkeiten von Demokratien zu erklären, haben insbesondere auf demokratiespezifische Institutionen und Entscheidungsverfahren verwiesen. ${ }^{3}$ Von zentraler Bedeutung sind in dieser Sichtweise regelmäßige freie Wahlen. Diese stellen nicht nur eine dem Mehrheitswillen entsprechende Auswahl der Regierung sicher. Noch wichtiger ist, dass das der Regierung unterstellte Interesse an einer Wiederwahl dazu führt, dass sie die Auswirkungen ihrer Politik auf die nächsten Wahlen antizipiert: »Because political leaders recognize the possibility of ex post punishment in the loss of office, ex ante they select policies they believe will be successful and hence lengthen their tenure« (Siverson 1995: 483).

Eine Schwäche dieses »electoral punishment model« besteht darin, dass der mögliche Verlust der nächsten Wahl nur für jene Entscheidungsträger abschreckend wirken kann, die erneut kandidieren können. Für zahlreiche Entscheidungsträger, denen nach der Verfassung ihres Landes eine erneute Wiederwahl untersagt ist (bspw. für den US-Präsidenten in seiner zweiten Amtszeit) wäre aus dieser Perspektive daher keine Zurückhaltung zu erwarten. Dem »electoral punishment model« stellen Dan Reiter und Allan Stam daher ein »contemporary consent model« entgegen, demzufolge Entscheidungsträger in Demokratien kontinuierlich um Unterstützung für ihre Sicherheitspolitik werben und »virtually never initiate war that is unpopular at the time« (Reiter/Stam 2002: 200).

Für diese kontinuierliche Rückbindung von Regierungshandeln an die öffentliche Meinung auch zwischen Wahlterminen kommt Parlamenten eine Schlüsselstellung zu. ${ }^{4}$ Zwar gibt es auch im Bereich der Sicherheitspolitik Interessengruppen und Nicht-

3 Eine alternative Erklärung hat anstelle von Institutionen die Bedeutung demokratiespezifischer Normen und politischer Kultur betont (vgl. Doyle 1983; Russett 1993; Owen 1994; Weart 1998). Aus dieser Perspektive versuchen Entscheidungsträger »to follow the same norms of conflict resolution as have been developed within and characterise their domestic political processes « (Russett 1993: 35). Da Politik in Demokratien durch friedliche Konfliktlösung geprägt ist, bevorzugen sie auch in der internationalen Politik Verhandlungen gegenüber dem Einsatz von Gewalt. Allerdings übersetzt sich diese Präferenz nur im Umgang mit anderen Demokratien in einen friedlichen Konfliktaustrag. In der Auseinandersetzung mit Nicht-Demokratien müssen Demokratien auf Strategien zurückgreifen, die den Einsatz von Gewalt nicht ausschließen, um das Risiko, selbst angegriffen zu werden, zu vermeiden (RisseKappen 1995). Kritiker verweisen darauf, dass dieser Ansatz nicht erklären kann, weshalb Demokratien anderen Demokratien durchaus mit dem Einsatz von Gewalt drohen (Layne 1994: 13) und weshalb sie zahlreiche Kolonialkriege geführt haben, die nicht der Selbstverteidigung, sondern der Unterwerfung anderer Völker dienten (Bueno de Mesquita et al. 1999; Rosato 2003: 588).

Versuche, den Demokratischen Frieden als Epiphänomen anderer Faktoren wie Machtrelationen (Layne 1994), Allianzen (Farber/Gowa 1995) oder ökonomischer Interdependenz (Gartzke 2007) zu erklären, haben sich hingegen nicht durchsetzen können.

4 Hänggi (2004: 11); vgl. auch Doyle (1983: 207); Chan (1984: 630); Morgan/Campbell (1991: 191); Auerswald (1999; 2001) und Elman (2000). 
Regierungsorganisationen, die Regierungshandeln an diejenigen zurückbinden helfen, die von Entscheidungen in besonderer Weise betroffen oder interessiert an ihnen sind. Das für die Vergesellschaftung des Regierens charakteristische Motiv, die Möglichkeiten politischer Steuerung durch die Einbindung der Regelungsadressaten zu verbessern, leidet im Bereich der Außenbeziehungen allerdings prinzipiell darunter, dass sich diese Adressaten außerhalb des politischen Systems befinden (Dembinski 2007). Aus diesem Grund rücken Parlamente umso mehr in den Mittelpunkt der demokratischen Kontrolle von Sicherheitspolitik jenseits von Wahlen.

Angesichts der Bedeutung, die die Konfliktforschung demokratischer Kontrolle im Allgemeinen und parlamentarischer Kontrolle im Besonderen für die Friedensneigung von Demokratien zumisst, fallen die erheblichen Unterschiede zwischen Demokratien in diesem Bereich auf. Als bspw. im Vorfeld des amerikanischen Angriffs auf den Irak 2003 in zahlreichen Demokratien über eine mögliche Beteiligung diskutiert wurde, hätten einige Regierungschefs nur nach vorheriger Zustimmung des Parlaments Truppen entsenden können, während andere bei dieser Entscheidung keinen institutionellen Beschränkungen unterworfen waren.

Trotz ihrer offenkundigen Bedeutung für das Konfliktverhalten von Staaten haben derartige institutionelle Unterschiede zwischen Demokratien aber bislang nur geringe Aufmerksamkeit erfahren. Sie sind zwar in einigen Arbeiten zur Erklärung unterschiedlicher Konfliktneigungen herangezogen worden: In einer empirischen Untersuchung zur (Nicht-)Beteiligung europäischer Demokratien am Irak-Krieg 2003 haben Sandra Dieterich, Hartwig Hummel und Stefan Marschall zeigen können, dass tatsächlich Demokratien mit einem hohen Maß an parlamentarischer Kontrolle signifikant weniger am Krieg beteiligt waren als Demokratien, in denen die Entscheidung von Regierungen ohne Beteiligung des Parlaments getroffen wurde (Dieterich et al. 2009; vgl. auch Auerswald 1999 und Elman 2000). Ungeklärt ist aber noch, worauf die unterschiedlichen Verfahren bei Entscheidungen über den Einsatz von Streitkräften überhaupt zurückzuführen sind. In diesem Beitrag befassen wir uns mit dieser Frage. Wir konzentrieren uns dabei auf jenen Ausschnitt demokratischer Entscheidungsverfahren, von dem ein besonders großer Einfluss auf das Konfliktverhalten von Demokratien zu erwarten ist, nämlich auf die An- bzw. Abwesenheit eines parlamentarischen Vetorechts beim Einsatz von Streitkräften: Warum genießt das Parlament in einigen Demokratien ein Vetorecht über den Einsatz des Militärs, während in anderen Demokratien die Regierung ohne Mitspracherecht des Parlaments entscheiden kann?

Unsere Untersuchung umfasst dabei Streitkräfteeinsätze in bewaffneten Konflikten mit anderen Staaten (wie etwa im Krieg gegen den Irak 2003), mit nicht-staatlichen Gewaltakteuren (wie etwa den Taliban) sowie in militärischen Interventionen zur Verhinderung eines Genozids (wie etwa im Kosovo), zur Absicherung eines Friedensabkommens (wie etwa in Bosnien seit 1995) oder zur Stabilisierung eines Staates nach einem Konflikt (wie in Afghanistan). Denn unabhängig von der Konfliktform und von völkerrechtlichen Grundlagen haben diese Einsätze gemein, dass sie das Leben von Soldaten bei der Verfolgung politischer Ziele riskieren und deshalb einer demokratischen Legitimation bedürfen (Lord 2008). Von unserer Untersuchung aus- 
genommen sind hingegen Militäreinsätze im Rahmen des Katastrophenschutzes und zur Unterstützung der Polizei im Innern.

Die Ursachen für die Existenz oder das Fehlen eines parlamentarischen Vetorechts bei solchen Militäreinsätzen wurden unseres Wissens noch nicht untersucht. Um Hypothesen über mögliche Ursachen zu generieren, wenden wir uns daher zwei Forschungsfeldern zu, die sich mit verwandten Fragen beschäftigen: zum einen der Konfliktforschung, soweit sie sich mit den Unterschieden in den Sicherheitspolitiken von Demokratien beschäftigt, zum anderen jenem Bereich der Vergleichenden Systemforschung, der sich generell mit der Stellung des Parlaments im politischen System auseinandersetzt (Abschnitt 2). Die so gewonnenen Hypothesen testen wir mit Hilfe statistischer Verfahren an einer eigens erstellten Datensammlung, die für 49 Demokratien im Zeitraum zwischen 1989 und 2004 Angaben zur An- bzw. Abwesenheit eines Parlamentsvorbehalts bei Militäreinsätzen enthält (Abschnitt 3). Wie wir zeigen können, ist die Abwesenheit eines Parlamentsvorbehalts dann wahrscheinlich, wenn sich ein Staat einem hohen Maß externer Bedrohung ausgesetzt sieht oder wenn es sich um einen Staat handelt, der in der britischen Verfassungstradition steht. Auch für den Einfluss vergangener Kriegserfahrungen finden wir Belege. Dagegen lässt sich nicht nachweisen, dass Transitionsdemokratien oder präsidentielle Systeme eine signifikant höhere Wahrscheinlichkeit für einen Parlamentsvorbehalt haben (Abschnitt 4).

\section{Erklärungsansätze}

Aus der Konfliktforschung lassen sich drei Hypothesen ableiten, die die Ursache parlamentarischer Vetomöglichkeiten bei Militäreinsätzen entweder in den Besonderheiten des Demokratisierungsprozesses (2.1), in den bisher gemachten sicherheitspolitischen Erfahrungen (2.2) oder in der internationalen Umwelt (2.3) verorten. Während die ersten beiden Hypothesen Akteursmerkmale betonen und sich damit in die Tradition liberaler Konfliktforschung stellen, handelt es sich bei der dritten Hypothese um eine »second image reversed«-Erklärung (Gourevitch 1978), die im Gegensatz zu second image-Erklärungen nicht postuliert, dass innerstaatliche Faktoren auf das internationale System einwirken, sondern dass umgekehrt Aspekte des internationalen Systems die Gestalt der innerstaatlichen Institutionen beeinflussen. ${ }^{5}$

Hinzu treten zwei Hypothesen aus der Vergleichenden Systemforschung, die die unterschiedliche Stellung des Parlaments in parlamentarischen und präsidentiellen Regierungssystemen sowie die einmalige Stellung des Parlaments in der britischen Verfassungstradition hervorheben (2.4).

5 Zur Unterscheidung der Analyseebenen Individuum (first image), Staat (second image) und internationales System (third image) bei der Analyse internationaler Beziehungen vgl. Waltz (1959). 


\subsection{Reduzierung von Unsicherheit: Die Demokratisierungs-Hypothese}

Die Demokratisierungs-Hypothese geht davon aus, dass während des Demokratisierungsprozesses ein besonders hoher Bedarf besteht, die Handlungsspielräume künftiger Regierungen einzuschränken. Im Gegensatz zu stabilen Demokratien ist in Staaten, die sich im Demokratisierungsprozess befinden, nicht nur unsicher, welche demokratische(n) Partei(en) künftig die Regierung bilden, sondern auch, ob sich die neuen demokratischen Eliten gegenüber den alten nicht-demokratischen Eliten überhaupt durchsetzen. Aus diesem besonders hohen Maß an Unsicherheit über die Akteure, die in Zukunft die exekutiven Positionen im Staat besetzen, resultieren besonders starke Anreize, deren Handlungsspielräume institutionell einzuschränken. Dies gilt gerade auch mit Blick auf Militäreinsätze, wie die überproportional häufige Verwicklung von Staaten im Demokratisierungsprozess in militärische Konflikte verdeutlicht (vgl. dazu Mansfield/Snyder 1995/96; 2005).

Eine verbreitete Strategie zur Einschränkung künftiger Handlungsspielräume ist die Delegation von Kompetenzen an internationale Organisationen. Tatsächlich konnten Edward Mansfield und Jon Pevehouse (2006; 2008) empirisch zeigen, dass Staaten im Demokratisierungsprozess generell überproportional häufig internationalen Organisationen beitreten. ${ }^{6}$ Eine alternative Strategie zur Einschränkung künftiger Handlungsspielräume der Regierung besteht in der Ausweitung der Kontrolle durch den wichtigsten institutionellen Gegenspieler, das Parlament. ${ }^{7}$ Zwar steht insbesondere in parlamentarischen Regierungssystemen der Regierung nicht das Parlament insgesamt als Kontrolleur gegenüber, weil Regierung und Regierungsmehrheit zu einer Funktionseinheit verschmolzen sind (King 1976). Dennoch bleiben Stärkungen des Parlaments selbst in parlamentarischen Systemen nicht wirkungslos, weil zum einen die Opposition als wichtigstes Kontrollorgan die Regierung leichter zur Rechenschaft ziehen kann und weil zum anderen die Hinterbänkler in den Regierungsfraktionen aufgewertet werden, die über parteiinterne Kanäle Einfluss auf die Regierung nehmen.

6 Am Beispiel der Europäischen Menschenrechtskommission (EMRK) hat auch Andrew Moravcsik (2000) zeigen können, dass Staaten im Demokratisierungsprozess in der Tat deutlich häufiger der EMRK beitreten als Diktaturen und stabile Demokratien. In diesen Staaten befinden sich zwar Demokraten an der Regierung, die die Menschenrechte einhalten. Allerdings gibt es nach wie vor starke anti-demokratische Kräfte und infolgedessen eine große Unsicherheit über die Menschenrechtspolitik nachfolgender Regierungen. Durch einen EMRKBeitritt kann die amtierende Regierung die Kosten für Menschenrechtsverletzungen für ihre Nachfolger erheblich erhöhen. Moravcsik zeigt, dass von dieser Gruppe die größte Nachfrage für einen EMRK-Beitritt ausgeht.

7 In der Transformationsforschung ist dem Parlament generell eine stabilisierende Wirkung für die Konsolidierung von Demokratie zugeschrieben worden. Vgl. hierfür insbesondere Linz (1990). 
Ein Parlamentsvorbehalt bei Militäreinsätzen ist aus dieser Sicht ein effektives Mittel, um Einsätze, die von den demokratischen Kräften abgelehnt werden, zu verhindern. Die Demokratisierungs-Hypothese lautet daher:

Wenn sich ein Staat noch im Transformationsprozess zu einer stabilen Demokratie befindet, ist das Vorhandensein eines Parlamentsvorbehalts bei Militäreinsätzen besonders wahrscheinlich.

\section{2. »Nie wieder Krieg«: Die Lessons-learnt-Hypothese}

Während die Demokratisierungs-Hypothese die Unsicherheit über zukünftiges Regierungshandeln in den Mittelpunkt stellt, betont die Lessons-learnt-Hypothese die bereits gemachten Erfahrungen mit vergangenem Regierungshandeln, insbesondere mit vorherigem Politikversagen. Bei der Bestimmung dessen, was Politikversagen im Bereich von Militäreinsätzen bedeutet, greifen wir auf die liberale Konfliktforschung zurück. Aus dieser Perspektive sind die Bürgerinnen und Bürger prinzipiell daran interessiert, die Kosten eines Militäreinsatzes zu vermeiden bzw. zu minimieren, während Regierungen eher zu militärischen Risiken bereit sind. ${ }^{8}$ Um diese Kriegsabneigung in Regierungspolitik zu übersetzen, bedarf es nicht unbedingt eines Parlamentsvorbehalts als besonderer institutioneller Schranke. Allerdings ist dessen Einführung immer dann wahrscheinlich, wenn zuvor die Regierung ihre Entscheidungsfreiheit nicht im Sinne der Bürgerschaft verwendet und diese in kostspielige und verlustreiche Militäreinsätze verwickelt hat. Mit der Demokratisierungs-Hypothese hat die Lessons-learnt-Hypothese gemein, dass sie die Schaffung von Institutionen bzw. die Ausweiterung ihrer Kompetenzen als kostspielig betrachtet und nur erwartet, wenn damit auf einen klar definierten Bedarf reagiert wird. In Anbetracht einer Regierung, die sicherheitspolitisch versagt und das Land in einen verlustreichen Krieg geführt hat, gibt es aus dieser Perspektive einen dringenden Bedarf nach verstärkter Kontrolle der Regierung, um eine Wiederholung des Politikversagens unwahrscheinlicher zu machen. Ein Parlamentsvorbehalt erscheint also umso wahrscheinlicher, je kostspieliger und verlustreicher vergangene Militäreinsätze gewesen sind. Dies deckt sich mit Erkenntnissen der policy-Forschung, dass Politikversagen immer auch ein

8 Dieser Topos findet sich bereits bei Immanuel Kant (1984) und wurde von der Demokratie-/ Frieden-Forschung aufgegriffen (Russett 1993). Allerdings hat die moderne Konfliktforschung den Kantschen Topos insofern modifiziert, als dass Kriege keineswegs unwahrscheinlich erscheinen, wenn sie zu geringen Kosten geführt werden können oder aber erhebliche Gewinne versprechen. Kritiker haben darüber hinaus auf Beispiele verwiesen, in denen Bürger und ihre Vertreter im Parlament kriegsbereiter waren als die Regierung (Elman 2000 mit Verweis auf Finnland im Zweiten Weltkrieg und Israel 1977-1981) oder in denen ein hohes Maß parlamentarischer Kontrolle die Kriegsführung behindert hat und infolgedessen eher das Parlament als Ursache von Politikversagen erscheint. Trotz dieser Modifikationen besitzt der Befund, dass die Popularität amtierender Regierungen mit der Zunahme von Gefallenen aus Militäreinsätzen abnimmt, weiterhin Gültigkeit (vgl. Mueller 1973; Eichenberg et al. 2006). Wir konzentrieren uns daher bei unserer Entwicklung der Lessons-learnt-Hypothese auf die Lesart der liberalen Konfliktforschung. 
Gelegenheitsfenster für umfassende Veränderungen, gerade auch im institutionellen Bereich, schafft (Hall 1993; Walsh 2007).

Für die Plausibilität dieser Hypothese spricht die Verabschiedung der »war powers resolution« durch den US-Kongress in der Endphase des Vietnam-Kriegs, die Einführung eines Parlamentsvorbehalts in Spanien nach der unpopulären Beteiligung am Irak-Krieg sowie die Parlamentsvorbehalte bei den beiden Hauptverlierern des Zweiten Weltkrieges, Deutschland und Japan. Die Lessons-learnt-Hypothese lautet:

Je größer das vergangene Politikversagen einer Regierung bei der Entsendung von Streitkräften, desto wahrscheinlicher die Existenz eines Parlamentsvorbehalts.

\subsection{Parlamentarische Kontrolle als Luxus? Die Bedrohungs-Hypothese}

Die Bedrohungs-Hypothese versteht innerstaatliche Entscheidungsprozesse als Resultat internationaler Einflüsse. Die Schwerfälligkeit und Transparenz, die liberale Theoretiker als Vorzug demokratischer Entscheidungsverfahren ansehen, sind aus Sicht der Bedrohungs-Hypothese dysfunktional, weil sie jene schnellen und flexiblen Reaktionen erschweren, die angesichts äußerer Bedrohungen als notwendig angesehen werden können. Die Bedrohungs-Hypothese knüpft somit an eine Tradition der Staats- und Demokratietheorie an, die die Außen- und Sicherheitspolitik als Privileg der Exekutive versteht und geringere Maßstäbe an ihre demokratische Kontrolle anlegt. ${ }^{9}$ Die Hypothese betont allerdings die Varianz äußerer Bedrohungen. Die Ausbildung demokratischer Entscheidungsverfahren wird demnach durch ein konfliktarmes sicherheitspolitisches Umfeld erleichtert bzw. durch ein bedrohliches Umfeld erschwert, denn »decentralizing power in the face of threat would seem inefficient and highly dangerous, perhaps even inviting attack" (Rasler/Thompson 2005: 44).

Die prominenteste Variante der Bedrohungs-Hypothese ist von Kritikern des Demokratischen Friedens im Kontext der so genannten reverse causality-Debatte vorgebracht worden. Danach ist ein friedliches internationales Umfeld nicht Folge, sondern Voraussetzung für Demokratie, während umgekehrt äußere Bedrohungen und Kriege zum Zusammenbruch demokratischer Verfahren führen können (vgl. Thompson 1996). Von Vertretern einer liberalen Erklärung des Demokratischen Friedens ist zwar vehement bestritten worden, dass der Friede zwischen Demokratien ein reines Epiphänomen internationaler Konfliktformationen sei. Durchaus anerkannt wird allerdings der grundsätzliche Zusammenhang, dass »international conflict, or an intense threat of conflict, [...] inimical to democracy« (Russett/Oneal 2001: 199) ist.

Die Bedrohungs-Hypothese knüpft an die Debatte über reverse causality an, tut dies jedoch in differenzierter Form. Die in der Debatte vorgebrachten Überlegungen über den Zusammenhang zwischen äußerer Bedrohung und innerstaatlichen Institutionen stehen noch in einer Tradition der Demokratie-Frieden-Debatte, welche die Dichotomie Demokratie-Autokratie als Bezugspunkt hat und ihr Augenmerk nicht

9 Vgl. Locke (1960, insbesondere $\S \S 145-148$ des »Second Treatise«) sowie de Tocqueville (1990: 234-235). 
auf die feinen Unterschiede zwischen Demokratien richtet. Jüngere Arbeiten plädieren jedoch dafür, über die Dichotomie zwischen Demokratie und Autokratie hinauszugehen. Die Auswirkungen internationaler Bedrohungen und Krisen auf Demokratien könnten in ihrer Komplexität nur verstanden werden, wenn nicht die demokratische Qualität eines Staates in seiner Gesamtheit in den Blick genommen werde. Vielmehr sei es wichtig, das Augenmerk darauf zu richten, wie sich das internationale Umfeld auf einzelne Dimensionen demokratischer Politik auswirke (vgl. Krebs 2009). Die Bedrohungs-Hypothese folgt diesem Ansatz, indem sie einen Zusammenhang zwischen äußerer Bedrohung und der An- oder Abwesenheit eines parlamentarischen Vetorechts herstellt. Sie lautet:

Je höher die externe Bedrohung, der eine Demokratie ausgesetzt ist, desto wahrscheinlicher ist das Fehlen eines parlamentarischen Vetorechts bei Militäreinsätzen.

\subsection{Spiegel des politischen Systems: Demokratietyp-Hypothesen}

Die bisherigen Hypothesen gehen alle von der Annahme aus, dass der Grad parlamentarischer Kontrolle von Entsendeentscheidungen durch Faktoren innerhalb des Politikfeldes Sicherheits- und Verteidigungspolitik bestimmt ist. Dagegen nehmen die aus der Vergleichenden Systemforschung gewonnenen Demokratietyp-Hypothesen an, dass sich der Grad parlamentarischer Kontrolle von Entsendeentscheidungen daraus ergibt, welche Rolle das Parlament generell im politischen System eines Landes spielt.

In diesem Zusammenhang ist die Unterscheidung zwischen parlamentarischem und präsidentiellem Regierungssystem von Bedeutung. Parlamentarische Systeme zeichnen sich durch die Abberufbarkeit der Regierung durch das Parlament aus (Steffani 1979: 39). Obwohl somit das Parlament im parlamentarischen Regierungssystem auf den ersten Blick eine besonders starke Stellung einnimmt, führt das Abberufungsrecht in der Regierungspraxis in aller Regel zu einer starken Verschränkung der Regierung mit der Mehrheit der sie unterstützenden Abgeordneten im Parlament, die durch starke Parteiorganisationen und einen daraus folgenden Fraktionszwang zusammengehalten werden. Die Kontrollfunktion des Parlaments wird daher in parlamentarischen und präsidentiellen Systemen auf unterschiedliche Weise wahrgenommen. In parlamentarischen Systemen kann die Regierungsmehrheit die Regierungspolitik stärker indirekt mitsteuern (Schwarzmeier 2001). Als Gegenspielerin der Regierung fungiert weniger das Parlament insgesamt als vielmehr die Opposition, die auf der Basis von Minderheitenrechten an der Kontrolle der Regierung mitwirkt. Ein formales Vetorecht für das Parlament über den exekutiven Einsatz militärischer Gewalt ist in der Funktionslogik eines solchen Systems nicht angelegt. Anders dagegen in präsidentiellen Systemen, in denen das Parlament als solches den Gegenpol zur Regierung darstellt. Ein verbrieftes parlamentarisches Vetorecht über den Einsatz von Streitkräften entspricht der Funktionslogik eines solchen Systems sehr viel eher. Vergleichende empirische Untersuchungen haben bestätigt, dass tatsächlich derartige explizite Kontrollmöglichkeiten von Parlamenten in präsidentiellen Regierungssystemen tenden- 
ziell größer sind als in parlamentarischen Systemen (Strøm 2000; Harfst/Schnapp 2003). Die Parlamentarismus-Hypothese lautet daher:

In parlamentarischen Systemen ist die Existenz eines parlamentarischen Vetorechts bei Militäreinsätzen unwahrscheinlicher als in Präsidialsystemen.

Eine weitere Demokratietyp-Hypothese geht von den Besonderheiten der britischen Verfassungstradition aus. Die Grundlinien der britischen Verfassung haben sich durch das britische Empire weit über die Grenzen Großbritanniens hinaus ausgewirkt und die Verfassungsgebung in vielen vormals abhängigen Gebieten nachhaltig beeinflusst. Dies ist für unseren Gegenstand umso bedeutsamer, als sich im britischen System früh im Prozess der Demokratisierung und Parlamentarisierung eine eigene Doktrin in Bezug auf die Rolle des Parlaments bei Militäreinsätzen entwickelt hat. Demnach ist der Einsatz der Streitkräfte Teil des »royal prerogative«, also ein Privileg der Exekutive, und bedarf keiner Zustimmung des Parlaments. ${ }^{10}$ Wenn die britische Tradition in den Verfassungen der vormals abhängigen Gebiete weiter wirkt, ist es wahrscheinlich, dass in diesen Staaten das Entscheidungsrecht über den Einsatz der Streitkräfte der Exekutive vorbehalten ist. Die Hypothese lautet daher:

In Ländern mit britischer Verfassungstradition ist die Existenz eines parlamentarischen Vetorechts bei Militäreinsätzen unwahrscheinlicher als in anderen Ländern.

\section{Forschungsdesign}

\subsection{Der PARLCON-Datensatz}

Um die An- bzw. Abwesenheit eines Parlamentsvorbehalts bei Streitkräfteeinsätzen erklären zu können, haben wir einen neuen Datensatz namens PARLCON erstellt, der die Jahre 1989-2004 umfasst. ${ }^{11}$ Dieser Zeitraum ist für die Untersuchung der Entscheidungsverfahren besonders interessant, weil die seit Ende des Ost-West-Konflikts verbreiteten Friedensmissionen und wars of choice weitaus größere Entscheidungsspielräume lassen als das zuvor dominante Szenario des Verteidigungsfalls. Berücksichtigt wurden nur Staaten, bei denen es sich in einem gegebenen Jahr ohne jeden Zweifel um eine Demokratie gehandelt hat, denn in autoritären oder eingeschränkt demokratischen Staaten wäre ein Parlamentsvorbehalt kaum aussagekräftig.

10 Albert Venn Dicey, zitiert nach White (2003: 300). Nigel White und Brendan Donnelly verdanken wir darüber hinaus den Hinweis, dass es Teil der britischen politischen Kultur sei, Regierungen zwischen Wahlen weitreichende Gestaltungsspielräume zu gewähren. Dafür wird die Regierung bei den nächsten Wahlen umso eindeutiger zur Verantwortung gezogen, da die Politik der abgelaufenen Regierungsperiode in ihrer ausschließlichen Verantwortung lag und in deutlich geringerem Maße auf komplexe Aushandlungsprozesse zurückging, die die politische Kultur in vielen anderen Staaten kennzeichnen, die nicht von der britischen Verfassungstradition geprägt wurden.

11 Eine ausführliche Beschreibung des Datensatzes sowie umfassende Darstellungen von Entsenderecht und -praxis aller untersuchten Staaten findet sich in Wagner et al. (2010). Für eine Diskussion der zahlreichen Varianten, in denen Demokratien in ihrem Entsenderecht die Anforderungen militärischer Effektivität und demokratischer Legitimation vermitteln vgl. außerdem Peters/Wagner i.E. Der Datensatz ist von den Autoren erhältlich. 
Aus diesem Grund wurden Staaten nur in jenen Jahren berücksichtigt, in denen ihr combined POLITY score des POLITY IV-Datensatzes die Höchstwerte für eine Demokratie von »9« oder »10« erreicht. ${ }^{12}$ Während einige Staaten diese Höchstwerte während des gesamten Untersuchungszeitraums aufweisen, sind andere Staaten dadurch teilweise nur mit wenigen Jahre vertreten, weil der Demokratisierungsprozess erst spät in die entsprechende Konsolidierungsphase eintritt oder aber die Errungenschaften dieses Prozesses nicht bewahrt werden können und ein Rückfall in un- oder semi-demokratische Strukturen stattfindet. Ganz aus der Untersuchung ausgeschlossen wurden Costa Rica, Mauritius und Panama, weil sie über keine eigenen Streitkräfte verfügen, sowie Taiwan, das international nur von wenigen Regierungen als Staat anerkannt ist und aus diesem Grunde bei Militäreinsätzen zwangsläufig eine Sonderstellung einnimmt. Insgesamt umfasst unsere Datensammlung damit 49 Demokratien über unterschiedlich lange Zeiträume bzw. 616 Staatenjahre. ${ }^{13}$

PARLCON ist eine dichotome Variable, die den Wert »1« erhält, wenn die Zustimmung des Parlaments vor einem Militäreinsatz erforderlich ist, und deren Wert » 0 « ist, wenn dies nicht der Fall ist. Ein Parlamentsvorbehalt gilt auch dann als vorhanden, wenn von einem generellen Parlamentsvorbehalt sehr kleine Ausnahmen vorgesehen sind. So sind bspw. in Irland Bagatelleinsätze von bis zu zwölf Soldaten ausgenommen. ${ }^{14}$ Wird hingegen eine bedeutsame Kategorie von Einsätzen ausgenommen, wie etwa bei einem NATO-Mitglied die Teilnahme an allen Arten von NATO-geführten Einsätzen, kann nicht mehr von einem Parlamentsvorbehalt die Rede sein.

Um die An- bzw. Abwesenheit eines Parlamentsvorbehalts zu bestimmen, haben wir sowohl die Rechtslage als auch die parlamentarische Praxis berücksichtigt. Letz-

12 Im POLITY-Projekt wird auf getrennten 11-Punkt-Skalen gemessen, inwieweit ein Staat demokratische und autokratische Merkmale aufweist. Durch die Subtraktion des Autokratie-Wertes vom Demokratie-Wert ergibt sich der combined POLITY score, der sich in der Konfliktforschung für die Bestimmung des Regimetyps durchgesetzt hat und der auch dieser Arbeit zugrunde liegt.

13 Verwendet wurde der POLITY IV-Datensatz in der Version 2006. Auf der Basis der POLITY-Werte werden 27 Staaten im gesamten Untersuchungszeitraum berücksichtigt. Die übrigen Staaten im Datensatz nehmen nur für kürzere Zeiträume einen combined POLITY score von » 9 « oder » 10 « an und werden entsprechend kürzer berücksichtigt. Für die Kodierung werden Regelungen in der Verfassung und besondere Entsendegesetze berücksichtigt. Eine tabellarische Übersicht des Datensatzes findet sich im Anhang. Für eine ausführliche Begründung der einzelnen Kodierungen vgl. Wagner et al. (2010).

14 In der Schweiz sind Einsätze von weniger als 100 Soldaten und weniger als drei Wochen Dauer von der Zustimmungspflicht ausgenommen, in Mazedonien rein humanitäre Operationen und in Schweden traditionelle peacekeeping-Operationen mit Zustimmung aller Konfliktparteien. In einigen Staaten kann die Regierung außerdem bei bestimmten Operationen und besonderer Dringlichkeit das Militär zunächst ohne Zustimmung entsenden, muss dann aber innerhalb einer bestimmten Frist die Zustimmung des Parlaments einholen oder das Militär zurückholen. Wir werten dies als Existenz eines parlamentarischen Vetorechts in Österreich (zwei Wochen Frist) und in Japan (20 Tage), In den USA existiert mit der war powers resolution eine vergleichbare Regel (60 Tage Frist), allerdings ist sie verfassungsrechtlich umstritten und wird in der Entsendepraxis nicht durchweg beachtet (Collier 1994: 55; Wagner et al. 2010: 90-92). Wir kodieren daher die USA durchgehend als Land, in dem keine vorherige Zustimmung des Parlaments zur Entsendung nötig ist (vgl. auch Born/Urscheler 2004: 63; Born/Hänggi 2005: 206). 
tere wurde auf der Basis von einschlägigen Länderstudien, von Zeitungsberichten über Entsendeentscheidungen und von Einschätzungen durch Länderexperten erhoben. In den allermeisten Fällen war die Rechtslage eindeutig und die Praxis im Einklang mit ihr. In wenigen Fällen war jedoch unklar, ob tatsächlich eine parlamentarische Zustimmung benötigt wird. So fehlt in einigen Fällen eine eindeutige Rechtsgrundlage und es hat sich keine allgemein anerkannte Praxis herausgebildet. In Italien gab es bspw. über den gesamten Untersuchungszeitraum keine Einigkeit darüber, ob die Zustimmung des Parlaments zur Entsendung des Militärs nötig ist oder nicht, und die Regierung wählte immer wieder andere rechtliche Instrumente zur Entsendung der Truppen, deren Zulässigkeit verfassungsrechtlich umstritten war (Brissa 2005). Auch in Deutschland war zu Beginn der 1990er Jahre umstritten, ob das Parlament Auslandseinsätzen der Bundeswehr zustimmen muss. Erst 1994 schuf das Bundesverfassungsgericht hier eine autoritative Interpretation der Verfassung, an der sich seitdem auch die politische Praxis ausrichtet. Dazu kommen Fälle, in denen ein Vetorecht nicht auf der Agenda stand, weil das betroffene Land generell keine bewaffneten Streitkräfte ins Ausland entsandt hat (wie zum Beispiel die Schweiz bis 2001 oder einige Transformationsstaaten unmittelbar nach dem Regimewechsel) oder in denen uns die nötigen Daten nicht zugänglich waren (Mongolei bis 2001). Insgesamt haben wir daher 51 Staatenjahre als »unbestimmbar« kodiert und von der folgenden Analyse ausnehmen müssen. ${ }^{15}$

Auffällig ist, dass sich die Regelungen über die Zustimmungspflicht des Parlaments zu Militäreinsätzen nur selten ändern. In 37 unserer 49 Staaten ist es im gesamten Untersuchungszeitraum bei derselben Regelung geblieben. Immerhin in zwölf dieser Staaten verfügt das Parlament über ein Vetorecht, in den übrigen 25 hingegen nicht. Substanzielle Veränderungen hat es nur in fünf Staaten gegeben: ${ }^{16}$ Während in Bulgarien, der Tschechischen Republik, Ungarn und der Slowakei der Parlamentsvorbehalt nach 2000 abgeschafft wurde, ${ }^{17}$ wurde er auf Zypern Ende 2003 eingeführt.

\subsection{Unabhängige Variablen}

Für die Operationalisierung der fünf Einflussfaktoren »Demokratisierung«, »Politikversagen «, »Bedrohung«, »parlamentarisches System« und »britische Verfassungstradition« können wir nur teilweise auf etablierte Indikatoren und vorhandene Da-

$15 \mathrm{Zu}$ den als »unbestimmbar« kodierten Staatenjahren gehören wegen umstrittener Rechtslage Deutschland (1989-1994), Italien (1989-2004) sowie Chile (2004); wegen fehlender Relevanz von Entsendefragen Südafrika (1994-1996), Litauen (1991-1992) und die Schweiz (1989-2001); sowie wegen fehlender Daten die Mongolei (1992-2001).

16 In den verbleibenden sieben Fällen waren entsprechende Regelungen entweder im gesamten Untersuchungszeitraum oder in einzelnen Jahren unbestimmbar.

17 Eine nähere Untersuchung dieser Fälle deutet darauf hin, dass diese Staaten während der Beitrittsverhandlungen zur NATO von dieser gedrängt wurden, NATO-geführte Militäreinsätze von vorhandenen Parlamentsvorbehalten auszunehmen. In den folgenden statistischen Untersuchungen bleibt dieser Faktor jedoch unberücksichtigt, weil aufgrund der geringen Fallzahl keine statistisch signifikanten Zusammenhänge nachgewiesen werden konnten. 
tensätze zurückgreifen, nämlich für die Bestimmung von Demokratien im Transformationsprozess und von parlamentarischen Systemen. Hingegen betreten wir mit der Operationalisierung von Politikversagen Neuland, und bei der Messung von äußerer Bedrohung schlagen wir eine Operationalisierung vor, die herkömmlichen Indikatoren unseres Erachtens überlegen ist.

\section{Demokratisierung und politisches System}

Für die Bestimmung von Demokratien, die sich noch im Transformationsprozess befinden, übernehmen wir die Operationalisierung von Mansfield und Pevehouse (2006; 2008). Eine Demokratie erhält demnach in einem Jahr den Wert »Transitionsstaat«, wenn sie erst seit weniger als fünf Jahren einen combined POLITY score, der höher als sechs ist, besitzt. Innerhalb unseres Samples sind Transitionsstaaten ein eher seltenes Phänomen. Es beinhaltet nur 12 Staaten, die sich zeitweise im Demokratisierungsprozess befinden. In keinem Jahr sind es mehr als acht, in den meisten Jahren weniger als drei.

Für die Identifizierung parlamentarischer Regierungssysteme greifen wir auf die Database of Political Institutions (DPI) zurück, die von der Weltbank erstellt wurde (Beck et al. 2001). Sie ist unseres Wissens die einzige Datenbank, die für alle von uns untersuchten Staaten Zeitreihen anbietet. Sie enthält eine »System«-Variable, welche die Werte »direkt präsidentiell«, »starker, von Versammlung gewählter Präsident« oder »parlamentarisch « annehmen kann. Weil nur wenige Staaten in die zweite Kategorie fallen, haben wir uns zur Dichotomisierung der Variablen durch Zusammenfassung der letzten beiden Kategorien entschieden. Unsere Dummy-Variable »parlamentarisches System« unterscheidet daher parlamentarische (einschließlich semipräsidentieller) von nicht-parlamentarischen Demokratien. ${ }^{18}$

Mit 35 Staaten dominieren parlamentarische Demokratien unser Sample. Dem stehen zwölf präsidentielle Demokratien gegenüber. Für zwei Staaten hält die DPI einen Wechsel des Regierungssystems fest: Die Mongolei ging 1994 von einem semi-präsidentiellen, Israel 1997 von einem parlamentarischen zu einem präsidentiellen System über. ${ }^{19}$

Als Indikator dafür, ob ein Land der britischen Verfassungstradition folgt, kann die Mitgliedschaft im Commonwealth of Nations gelten. ${ }^{20}$ Mitglieder des Commonwealth

18 In wenigen Grenzfällen können die Kodierungsentscheidungen der DPI-Autoren in Frage gestellt werden. Wir haben uns dennoch gegen jeglichen Eingriff in die Datenbank entschieden, weil nur die vollständige Übernahme des gesamten Datensatzes den Verdacht ausräumen kann, Korrekturen seien - bewusst oder unbewusst - mit Blick auf den postulierten Zusammenhang zwischen Regierungssystem und parlamentarischem Vetorecht bei Militäreinsätzen erfolgt.

19 Außerdem wechselten Ungarn 1991 und Bulgarien 2001 von einem semi-präsidentiellen zu einem parlamentarischen System, was aber unsere Kodierung nicht verändert.

20 Die Wahl dieses Indikators führt zum Ausschluss Irlands von dieser Gruppe, was unseres Erachtens allerdings für die Validität des Indikators spricht, da sich die irische Verfassung tatsächlich in vielerlei Hinsicht weit weniger am britischen Vorbild orientiert als die anderer Staaten. 
of Nations sind neben dem Vereinigten Königreich Australien, Botswana, Kanada, Indien, Jamaica, Neuseeland, Papua-Neuguinea, Südafrika ${ }^{21}$, Trinidad und Tobago und Zypern.

\section{Politikversagen}

Während bei allen bisherigen Operationalisierungen eine Dichotomisierung sinnvoll erschien, bietet sich für die Messung von Politikversagen eine Intervallskalierung an. Ausgangspunkt ist der Befund der Konfliktforschung, dass ein Militäreinsatz umso mehr als gescheitert gilt, je höher die Zahl der eigenen Gefallenen ist. Zur Messung greifen wir auf die Daten des Correlates-of-War-Projekts zurück. Zunächst entnehmen wir dem Interstate War Participants Dataset die Zahl eigener Gefallener aus allen zwischenstaatlichen Kriegen seit Beginn des Zweiten Weltkriegs. ${ }^{22}$ Diese setzen wir dann zur Bevölkerungszahl zu Kriegsbeginn ins Verhältnis. Allerdings können Gefallene je nach Kontext in unterschiedlichem Ausmaß Regierungsversagen anzeigen. So steht zu vermuten, dass Gefallene in verlorenen Kriegen stärker ins Gewicht fallen als Gefallene in Kriegen, die gewonnen wurden. Zudem ist nicht auszuschließen, dass Gefallene in Kriegen, die die Regierung selbst initiiert hat, bedeutsamer sind als solche in Verteidigungskriegen. Die mögliche Bedeutung von Initiierung und Kriegsausgang berücksichtigen wir, indem wir neben der ungewichteten Fassung für die Variable verschiedene Varianten berechnen, in denen die Zahl der Gefallenen aus selbst initiierten und/oder verlorenen Kriegen jeweils mit dem Faktor 100 multipli-

21 Südafrika wurde erst nach dem Ende der Apartheid 1994 in das Commonwealth aufgenommen. In unserem Datensatz wird Südafrika ebenfalls 1994 zum ersten Mal berücksichtigt.

22 Da diese Datenbank 1997 endet, haben wir für den Zeitraum 1998 bis 2004 Daten ergänzt. Mit Hilfe des UCDP/PRIO Armed Conflict Dataset (vgl. Harbom/Wallensteen 2007) haben wir den Kargil-Krieg zwischen Indien und Pakistan 1999 und den Irakkrieg 2003 hinzugefügt. Daten zu Gefallenenzahlen haben wir zum einen Ganguly (2001: 117), zum anderen dem Iraq Coalition Casualties Count (http://icasualties.org; 30.11.2009) entnommen.

In einer alternativen Analyse haben wir außerdem die Daten des COW Extrastate War Participants Dataset berücksichtigt. Dies enthält bis 1997 Daten über die Kriege, die Staaten gegen nicht-staatliche Akteure geführt haben, wozu in erster Linie die Dekolonisierungskriege zählen. Dies führt aber zu keiner erkennbaren Veränderung unserer Ergebnisse, da die Werte der Variable Politikversagen mit und ohne Berücksichtigung der »extrastate wars « nahezu identisch sind. In jedem Jahr beträgt der Korrelationskoeffizient für die Korrelation zwischen den beiden Berechnungsarten 1,0. Wir haben uns daher entschieden, auf die Berücksichtigung der »extrastate wars« zu verzichten, zumal im Falle verlustreicher und verlorener Kolonialkriege die naheliegendste Lehre aus Politikversagen nicht die Reform der Entscheidungsmechanismen ist, sondern die Entlassung der ehemaligen Kolonien in die Unabhängigkeit (für Frankreich während und nach dem AlgerienKrieg vgl. Krebs 2010). 
ziert wird. ${ }^{23}$ Für alle Varianten folgen wir außerdem dem Vorschlag von Giacomo Chiozza und Hein Goemans (2004), die Daten hyperbolisch zu transformieren, um dem abnehmendem Effekt über Zeit gerecht zu werden, das heißt wir dividieren die (gewichtete) Gefallenenzahl durch die Zahl der Jahre seit dem Ende eines Krieges. Dadurch nehmen die Werte für das Politikversagen einer Regierung umso mehr ab, je weiter ein Konflikt zurückliegt. Gleichzeitig bleibt die Wirkung von verlustreichen Kriegen weit über das Konfliktende hinaus spürbar. Durch die hyperbolische Transformation unserer Daten können wir so darstellen, dass sich im unmittelbaren Anschluss an Politikversagen ein Gelegenheitsfenster für institutionelle Reformen öffnet, das sich allerdings in der Folgezeit mit abnehmender Geschwindigkeit wieder schließt.

\section{Bedrohung}

Für die Messung äußerer Bedrohung erscheinen vorhandene Indikatoren als nur wenig hilfreich. Traditionell diente die Zahl an Nachbarstaaten als Indikator für das Ausmaß äußerer Bedrohung (vgl. bspw. Midlarsky 1995). Diese Operationalisierung knüpft an den Befund der quantitativen Kriegsursachenforschung an, dass angrenzende Staaten mit größerer Wahrscheinlichkeit Krieg führen als andere Staatenpaare (vgl. dazu Bremer 1992). Insbesondere für den hier interessierenden Untersuchungszeitraum erscheint diese Operationalisierung allerdings aus mindestens zwei Gründen unangemessen. Erstens ist die bloße Anzahl angrenzender Staaten weit weniger bedeutsam als deren Absichten und militärische Möglichkeiten. Zweitens ist gerade die Zeitperiode seit dem Ende des Ost-West-Konflikts durch eine Entgrenzung der militärischen Bedrohungen charakterisiert.

In unserer Untersuchung haben wir daher zwei alternative Indikatoren für das Maß der äußeren Bedrohung verwendet: die Zahl vergangener militarisierter Konflikte und die Höhe der Verteidigungsausgaben. Die Verwendung zweier, sich ergänzender Indikatoren erscheint gerade bei der Bestimmung von Bedrohungen sinnvoll, weil sich diese definitionsgemäß auf Unbekanntes und Unwägbares beziehen und deshalb objektivierbaren Messungen besonders schwer zugänglich sind.

Die Zahl militarisierter Konflikte (MIDs), ${ }^{24}$ in die ein Staat verwickelt war, zeigt das tatsächliche Ausmaß militärischer Gewalt, das ein Staat im Umgang mit anderen - angrenzenden oder weiter entfernten - Staaten erfahren hat. Die Häufigkeit militarisierter Dispute ist ein Indikator für die Konflikthaftigkeit des Sicherheitsumfelds und für die Wahrscheinlichkeit, in militärische Auseinandersetzungen verwickelt zu werden. Die Häufigkeit zurückliegender militarisierter Konflikte ist dabei immer auch

23 Wiederum müssen wir die Daten für den Kargil- und den Irak-Krieg ergänzen. Für den Kargil-Krieg kodieren wir Indien als Initiator und den Ausgang als unentschieden (vgl. Ganguly 2001; Rothermund 2002). Bei der Kodierung des Irakkrieges (mit Stand 2004!) folgen wir dem UCDP/PRIO Armed Conflict Dataset, das die Koalitionstruppen als Initiator und Gewinner führt.

24 Zur Definition vgl. Gochman/Maoz (1984). 
ein Indikator für das aktuelle Maß äußerer Bedrohung. Allerdings prägen vergangene MIDs das aktuelle Bedrohungsmaß umso weniger, je länger diese zurückliegen. Deshalb weisen wir jedem MID im stattfindenden Jahr den Wert $» 1$ « zu und transformieren diesen Wert für die folgenden Jahre wiederum hyperbolisch, indem wir ihn durch die Zahl der seit Ende des Konflikts vergangenen Jahre dividieren. Der MIDDatensatz erfasst nur den Zeitraum bis 2001, weshalb keine Werte für die Jahre 2002 bis 2004 errechnet werden können.

Definitionsgemäß beziehen sich Bedrohungen nicht nur auf bereits militarisierte Konflikte, sondern darüber hinaus auf das schwer zu kalkulierende Risiko einer Militarisierung latenter Konflikte. Für diese Einschätzung äußerer Bedrohung erscheinen die Verteidigungsausgaben als geeigneter Indikator. Natürlich müssen die Verteidigungsausgaben zum Bruttonationalprodukt eines Staates in Beziehung gesetzt werden, um Vergleichbarkeit zu gewährleisten. Um Verzerrungen durch kurzfristige Schwankungen zu vermeiden, nehmen wir für jedes Staatenjahr das Dreijahresmittel aus dem betroffenen Jahr und den beiden vorangegangenen Jahren.

Für den von uns untersuchten Zeitraum kommen grundsätzlich zwei Datensammlungen in Frage, um die Verteidigungsausgaben zu erfassen: die jährlich in der »Military Balance« veröffentlichten Daten des International Institute for Strategic Studies (IISS) und die ebenfalls jährlich aktualisierten Daten des Stockholm International Peace Research Institute (SIPRI). Die Daten des SIPRI gelten gemeinhin als zuverlässiger und haben weitere Verbreitung in der Literatur. Sie weisen für unsere Zwecke den Nachteil auf, dass nicht für alle in PARLCON vorhandenen Länder und Jahre Daten vorhanden sind, ${ }^{25}$ wodurch der bei den geringen Fallzahlen ohnehin schon schwierige Nachweis statistisch signifikanter Zusammenhänge weiter erschwert wird. Wir führen daher getrennte Auswertungen auf der Basis beider Datenquellen durch.

Alles in allem erscheint gerade für das schwer zu messende Konzept der äußeren Bedrohung die Verwendung zweier sich ergänzender Indikatoren sinnvoll. Während die ausschließliche Verwendung des MID-Indikators die Schwäche hätte, alle nicht bereits militarisierten Konflikte ignorieren zu müssen, hätte die ausschließliche Verwendung des Verteidigungsausgaben-Indikators den Nachteil, lediglich regierungsamtliche Bedrohungsperzeptionen zu erfassen, von denen noch dazu nicht vollständig ausgeschlossen werden kann, dass sie neben sicherheitspolitischen auch rüstungspolitische Zielsetzungen verfolgen. Darüber hinaus könnte bei ausschließlicher Verwendung des MID-Indikators eingewandt werden, dass die Häufigkeit militarisierter Konflikte nicht Ursache, sondern Ergebnis parlamentarischer Kontrolle ist. Eine solches reverse causality-Argument kann für den Verteidigungsausgaben-Indikator nicht gemacht werden, weil alle hier untersuchten Parlamente unabhängig von der An- oder Abwesenheit eines Vetos bei Militäreinsätzen die Letztentscheidung über die Verteidigungsausgaben besitzen.

25 Betroffen sind für das Dreijahresmittel 68 Staatenjahre gegenüber drei Staatenjahren bei den IISS-Daten. 


\section{Untersuchungsergebnisse}

Welche Zusammenhänge gibt es zwischen den oben eingeführten Variablen und der An- oder Abwesenheit eines parlamentarischen Vetorechts bei der Entsendung des Militärs? Wir untersuchen den PARLCON-Datensatz auf einschlägige Muster, indem wir die Mittelwerte der verschiedenen Variablen zwischen den beiden Gruppen von Ländern mit und ohne parlamentarisches Vetorecht vergleichen. Dabei wird sich zeigen, dass Staaten ohne parlamentarisches Vetorecht vor allem in dreierlei Hinsicht deutlich (und statistisch signifikant) von Ländern mit Vetorecht abweichen. Sie blicken im Mittel auf verlustreichere verlorene Kriege zurück, sehen sich im Mittel stärkeren Bedrohungen ausgesetzt und zählen überdurchschnittlich häufig zur Gruppe der Commonwealth-Staaten.

Generell erschwert wird die Auswertung durch zwei Probleme: die starke Autokorrelation in unserem Datensatz und die geringe Fallzahl. Die Autokorrelation macht eine Zeitreihenanalyse wenig ergiebig. Da sich die Vetokompetenzen des Parlaments überhaupt nur in fünf Ländern substanziell über Zeit verändern, verspricht eine statistische Analyse solcher diachronen Veränderungen keinen Gewinn. Wir konzentrieren uns daher darauf, synchron die Unterschiede zwischen den Ländern zu untersuchen. Dazu analysieren wir unseren Datensatz für alle sechzehn Jahre getrennt und nutzen die Ergebnisreihen, um die Robustheit der Ergebnisse unseres Ländervergleichs über Zeit abzuschätzen. Um die Auswertung nicht allzu unübersichtlich werden zu lassen, berichten wir die Ergebnisse nur für jedes fünfte Jahr (also 1989, 1994, 1999, 2004) und fassen die Auswertungen der übrigen Jahre in den Anmerkungen zusammen.

Daraus ergibt sich als zweites Problem allerdings eine sehr niedrige Fallzahl. Die absoluten Fallzahlen variieren zwischen 28 und 42 Staaten (1989 vs. 2002). Das erlaubt es uns nur sehr eingeschränkt, multivariate Analysen durchzuführen, also die Wirkung aller potentiellen unabhängigen Variablen auf die Existenz eines parlamentarischen Vetorechts gleichzeitig zu analysieren. Zwar gibt es keine festen Fallzahlgrenzen für die Durchführung einer multiplen logistischen Regression. Aber in der Literatur werden gemeinhin Richtwerte angegeben, die von unseren Daten nicht erreicht werden. Ausschlaggebend ist dabei weniger die Fallzahl insgesamt als vielmehr die Zahl der »Ereignisse«, also in unserem Zusammenhang: die Zahl der Länder mit parlamentarischem Vetorecht. Peter Peduzzi et al. (1996) empfehlen generell nicht weniger als zehn Ereignisse pro unabhängiger Variable für die Durchführung einer multiplen logistischen Regression. Eric Vittinghoff und Charles McCulloch (2007) haben dieses Kriterium jüngst etwas zurückgenommen und argumentiert, dass zwar bei einer Zahl von zwei bis vier Ereignissen pro unabhängiger Variable verzerrte Ergebnisse wahrscheinlich sind, die Fehler bei einer größeren Zahl von Ereignissen pro unabhängiger Variable jedoch vertretbar sein können. Unsere maximale Zahl von Ländern mit parlamentarischem Vetorecht liegt bei 16 (2002), d.h. selbst unter günstigen Umständen können wir mit maximal drei unabhängigen Variablen in einer logistischen Regression arbeiten. 
Wir beschränken uns daher im Kern auf die Auswertung der bivariaten Beziehungen, zwischen den einzelnen unabhängigen Variablen und unserem Explanandum, der Existenz eines parlamentarischen Vetorechts über Militäreinsätze. Da eine ausschließliche Betrachtung bivariater Zusammenhänge Scheinkorrelationen nicht vollständig ausschließen kann, berichten wir abschließend kurz auch die Ergebnisse einer multiplen logistischen Regression für jene Jahre, in denen die Zahl der Länder mit parlamentarischem Vetorecht größer als zwölf ist.

\subsection{Demokratisierungs-Hypothese}

Die Überprüfung der Demokratisierungs-Hypothese wird zunächst durch die geringe Zahl von Transitionsdemokratien in unserem Sample erschwert. Der Anteil dieser Staaten beträgt in den meisten Jahren weniger als zwölf Prozent und steigt nur 1993/94 kurz auf etwa $18 \%$ an. In Tabelle 1 ist zu sehen, dass Transitionsstaaten über die Jahre hinweg in beiden Gruppen, also bei den Staaten mit und bei jenen ohne Parlamentsveto, ungefähr gleich häufig oder besser: gleich selten vorkommen. Systematische Unterschiede sind nicht zu erkennen. Der erwartete Effekt der Demokratisierung lässt sich also nicht beobachten. Dies mag aber nicht zuletzt den geringen Fallzahlen geschuldet sein.

\section{Tabelle 1: Verteilung der Transitionsdemokratien; zwei-seitige Signifikanztests}

\begin{tabular}{|l|rr|rr|rr|rr|}
\hline Jahr & \multicolumn{2}{|c|}{1989} & \multicolumn{2}{|c|}{1994} & \multicolumn{2}{|c|}{1999} & \multicolumn{2}{|c|}{2004} \\
\hline Parl. Vetorecht vor Militäreinsätzen & ja & nein & ja & nein & ja & nein & ja & nein \\
\hline N Transitionsdemokratien/gesamt & $1 / 11$ & $0 / 17$ & $3 / 12$ & $3 / 21$ & $0 / 14$ & $0 / 23$ & $1 / 13$ & $1 / 28$ \\
Anteil Transitionsdemokratien & $\mathbf{0 , 0 9}$ & $\mathbf{0}$ & $\mathbf{0 , 2 5}$ & $\mathbf{0 , 1 4}$ & $\mathbf{0}$ & $\mathbf{0}$ & $\mathbf{0 , 0 8}$ & $\mathbf{0 , 0 4}$ \\
Exakter Test nach Fisher & & $\mathrm{p}=0,393$ & & $\mathrm{p}=0,643$ & & $\mathrm{n} / \mathrm{a}$ & $\mathrm{p}=0,539$ \\
\hline
\end{tabular}

Um das Fallzahlproblem zu entschärfen, testen wir außerdem eine erweiterte Version der Demokratisierungs-Hypothese, die nicht dichotome Transitionsstaaten von anderen Demokratien unterscheidet, sondern generell auf das Alter der Demokratien fokussiert. Angesichts der besonderen Bedeutung parlamentarischer Kontrollverfahren für Staaten im Transitionsprozess sollten in jungen Demokratien vorwiegend starke Parlamentsrechte, wie ein Veto über Militäreinsätze, institutionalisiert sein. In etablierten, älteren Demokratien dagegen sind starke Parlamentsrechte weniger wichtig und sollten demnach weniger häufig zu beobachten sein. Alles in allem lässt sich also in Anlehnung an die Demokratisierungs-Hypothese ein Zusammenhang zwischen dem Alter einer Demokratie und der Existenz eines parlamentarischen Vetos vermuten. ${ }^{26}$

26 Wir danken einem anonymen Gutachter für diesen Hinweis. 
Um diese Vermutung zu überprüfen, messen wir für jeden Staat die Zahl der Jahre, die vergangen sind, seit er zum letzten Mal den Schwellenwert »6« für eine Demokratie beim combined POLITY score überschritten hat. Tatsächlich lässt sich feststellen, dass Staaten mit parlamentarischem Veto über Militäreinsätze fast durchweg im Mittel jüngere Demokratien sind als jene ohne Parlamentsveto (Tabelle 2). Allerdings ist die Streuung in beiden Gruppen recht groß und in den Jahren 2003 und 2004 kehrt sich das Verhältnis zwischen beiden Gruppen um. Zudem ist der Unterschied in keinem Jahr statistisch signifikant, so dass auch diese Lesart der Hypothese sich nicht bestätigt.

Tabelle 2: Mittelwertvergleich für das Alter der Demokratien mit und ohne parlamentarisches Vetorecht. Angegeben sind die Mittelwerte in Jahren, Standardabweichung in Klammern, zweiseitige Signifikanztests unter der Annahme gleicher Varianzen (geprüft mit Levenes Test).

\begin{tabular}{|l|rr|rr|rr|rr|}
\hline Jahr & \multicolumn{2}{|c|}{1989} & \multicolumn{2}{|c|}{1994} & \multicolumn{2}{|c|}{1999} & \multicolumn{2}{|c|}{$\mathbf{2 0 0 4}$} \\
\hline Parl. Vetorecht vor Militäreinsätzen & ja & nein & ja & nein & ja & nein & ja & nein \\
\hline N & 11 & 17 & 12 & 21 & 14 & 23 & 13 & 28 \\
\hline \multirow{2}{*}{ Alter der Demokratie } & $\mathbf{3 2 , 8}$ & $\mathbf{5 3 , 5}$ & $\mathbf{3 2 , 5}$ & $\mathbf{4 9 , 5}$ & $\mathbf{3 6 , 4}$ & $\mathbf{5 1 , 6}$ & $\mathbf{5 4 , 5}$ & $\mathbf{4 7 , 1}$ \\
& $(24,2)$ & $(47,9)$ & $(27,3)$ & $(47,4)$ & $(26,5)$ & $(46,5)$ & $(39,8)$ & $(46,4)$ \\
T-Test & & $\mathrm{p}=0,197$ & & $\mathrm{p}=0,266$ & & $\mathrm{p}=0,271$ & & $\mathrm{p}=0,623$ \\
\hline
\end{tabular}

\subsection{Lessons-learnt-Hypothese}

Die Lessons-learnt-Hypothese schneidet auf den ersten Blick ebenso wenig erfolgreich ab (Tabelle 3). Die Kennzahlen für das Politikversagen unterscheiden sich zwar in allen Varianten in allen Jahren zwischen den Ländern mit parlamentarischem Vetorecht und jenen ohne. Fast durchweg zeigen dabei Staaten mit parlamentarischem Vetorecht im Mittel die erwarteten höheren Werte. Vor allem nach 1994, nachdem also Deutschland in die Analyse miteinbezogen wird, ist dieser Unterschied deutlich erkennbar. Allerdings sind die Unterschiede nur in einer Variante tatsächlich statistisch signifikant, nämlich dann wenn die Gefallenen, die ein Land in einem verlorenen Krieg hinnehmen musste, besonders stark gewichtet werden. Dies gilt für alle Jahre, nicht nur die in Tabelle 3 berichteten, von 1990 bis 2003. Die Gewichtung der selbst initiierten Kriege hingegen erzeugt keine statistisch signifikanten Unterschiede zwischen den Gruppen und auch in der ungewichteten Variante sind keine signifikanten Unterschiede zwischen der Gruppe mit und jener ohne parlamentarisches Vetorecht zu beobachten.

Es gibt also Anzeichen dafür, dass in Ländern, in denen in der Vergangenheit verlustreiche Kriege verloren wurden, eher ein parlamentarisches Vetorecht existiert als in anderen Demokratien. Herausragende Beispiele aus dem Sample sind Deutschland, 
Japan, Finnland und Ungarn, die die höchsten Gefallenenzahlen für verlorene Kriege aufweisen und alle über ein Parlamentsveto verfügen. ${ }^{27}$

Tabelle 3: Mittelwertvergleich für die Variable Politikversagen (mit unterschiedlichen Gewichtungen für die Zahl der Gefallenen) für Länder mit und ohne parlamentarisches Vetorecht. Angegeben sind die Mittelwerte, Standardabweichung in Klammern, zweiseitige Signifikanztests unter der Annahme ungleicher Varianzen (geprüft mit Levenes Test).

\begin{tabular}{|c|c|c|c|c|c|c|c|c|}
\hline Jahr & \multicolumn{2}{|c|}{1989} & \multicolumn{2}{|c|}{1994} & \multicolumn{2}{|c|}{1999} & \multicolumn{2}{|c|}{2004} \\
\hline Parl. Vetorecht vor Militäreinsätzen & ja & nein & ja & nein & ja & nein & ja & nein \\
\hline $\mathrm{N}$ & 11 & 17 & 12 & 21 & 14 & 23 & 13 & 28 \\
\hline $\begin{array}{l}\text { Kennzahl Politikversagen, ohne } \\
\text { Gewichtung der Gefallenenzahlen } \\
\text { T-Test }\end{array}$ & $\begin{array}{r}0,10 \\
(0,21)\end{array}$ & $\begin{array}{r}\mathbf{0 , 0 7} \\
(\mathbf{0 , 0 7}) \\
p=0,671\end{array}$ & $\begin{array}{r}0,11 \\
(0,20)\end{array}$ & $\begin{array}{r}0,07 \\
(0,09) \\
p=0,518\end{array}$ & $\begin{array}{r}0,14 \\
(0,26)\end{array}$ & $\begin{array}{r}\mathbf{0 , 0 6} \\
(\mathbf{0 , 0 8}) \\
\mathrm{p}=0,286\end{array}$ & $\begin{array}{r}0,14 \\
(0,24)\end{array}$ & $\begin{array}{r}0,07 \\
(0,09) \\
p=0,305\end{array}$ \\
\hline $\begin{array}{l}\text { Kennzahl Politikversagen, Gefallene } \\
\text { in verlorenen Kriegen gewichtet } \\
\text { T-Test }\end{array}$ & $\begin{array}{r}9,6 \\
(21,5)\end{array}$ & $\begin{array}{r}\mathbf{0 , 2} \\
(\mathbf{0 , 5}) \\
p=0,176\end{array}$ & $\begin{array}{r}10,6 \\
(19,5)\end{array}$ & $\begin{array}{r}0,2 \\
(0,3) \\
p=0,092\end{array}$ & $\begin{array}{r}14,0 \\
(25,5)\end{array}$ & $\begin{array}{r}0,1 \\
(0,3) \\
\mathrm{p}=0,062\end{array}$ & $\begin{array}{r}11,9 \\
(24,0)\end{array}$ & $\begin{array}{r}2,1 \\
(6,9) \\
p=0,171\end{array}$ \\
\hline $\begin{array}{l}\text { Kennzahl Politikversagen, Gefallene } \\
\text { in initiierten Kriegen gewichtet } \\
\text { T-Test }\end{array}$ & $\begin{array}{r}0,1 \\
(0,4)\end{array}$ & $\begin{array}{r}\mathbf{0 , 3} \\
(\mathbf{0 , 8}) \\
p=0,400\end{array}$ & $\begin{array}{r}0,1 \\
(0,3)\end{array}$ & $\begin{array}{r}\mathbf{0 , 2} \\
(\mathbf{0 , 6 )} \\
\mathrm{p}=0,558\end{array}$ & $\begin{array}{r}5,9 \\
(21,8)\end{array}$ & $\begin{array}{r}0,2 \\
(0,4) \\
p=0,342\end{array}$ & $\begin{array}{r}5,9 \\
(20,7)\end{array}$ & $\begin{array}{r}\mathbf{0 , 2} \\
(\mathbf{0 , 4 )} \\
p=0,340\end{array}$ \\
\hline $\begin{array}{l}\text { Kennzahl Politikversagen, Gefallene } \\
\text { in verlorenen und initiierten Kriegen } \\
\text { gewichtet } \\
\text { T-Test }\end{array}$ & $\begin{array}{r}14,7 \\
(35,3)\end{array}$ & $\begin{array}{r}11,3 \\
(45,3) \\
p=0,825\end{array}$ & $\begin{array}{r}14,8 \\
(30,9)\end{array}$ & $\begin{array}{r}7,0 \\
(31,1) \\
p=0,494\end{array}$ & $\begin{array}{r}594,8 \\
(2179,2)\end{array}$ & $\begin{array}{r}5,3 \\
(24,0) \\
p=0,330\end{array}$ & $\begin{array}{r}584,3 \\
(2070,1)\end{array}$ & $\begin{array}{r}5,6 \\
(19,1) \\
p=0,333\end{array}$ \\
\hline
\end{tabular}

\subsection{Bedrohungs-Hypothese}

Die äußere Bedrohung messen wir mit zwei Indikatoren und bei beiden sind die Unterschiede zwischen den Gruppen klar erkennbar (Tabelle 4). Staaten, in denen das Parlament ein Veto über den Einsatz des Militärs besitzt, haben im Mittel niedrigere Militärausgaben und sind seltener in militärische Konflikte verwickelt. Besonders klar nachweisen lässt sich der Zusammenhang bei den Verteidigungsausgaben. Die Unterschiede zwischen Staaten mit und ohne parlamentarisches Vetorecht sind klar erkennbar und weisen in die erwartete Richtung, unabhängig davon, ob die Zahlen des SIPRI oder des IISS zu Grunde gelegt werden. Bei den Zahlen des SIPRI, die in der Literatur gemeinhin als zuverlässiger eingestuft werden, sind die Unterschiede

27 Außer Ungarn im Jahr 2004. 
zwischen den Staatengruppen in 12 der 16 untersuchten Jahre statistisch signifikant, bei den IISS-Zahlen immerhin noch in sieben Jahren. ${ }^{28}$

Auch im Hinblick auf die Beteiligung an MIDs unterscheiden sich Staaten mit und ohne parlamentarisches Vetorecht wie erwartet, allerdings sind die Unterschiede nur in 5 der 13 Jahre, für die Daten vorliegen, statistisch signifikant. ${ }^{29}$ Zwar könnte man vermuten, dass die Ergebnisse durch die Existenz von zwei Extremfällen verzerrt sind. So verfügt Israel über besonders hohe Verteidigungsausgaben, deren Anteil am Bruttonationalprodukt in jedem Jahr rund doppelt so hoch wie im jeweils folgenden Staat ist. Außerdem liegen die Kennzahlen der USA für die Beteiligung an MIDs mehr als doppelt so hoch wie die des folgenden Staates. Doch selbst wenn man diese Extremfälle aus der Untersuchung ausschließt, bleiben die Unterschiede zwischen Staaten mit und ohne parlamentarisches Vetorecht deutlich sichtbar und statistisch signifikant.

Tabelle 4: Mittelwertvergleich für die Bedrohungsindikatoren für Länder mit und ohne parlamentarisches Vetorecht. Angegeben sind die Mittelwerte, Standardabweichung in Klammern, zweiseitige Signifikanztests ${ }^{30}$

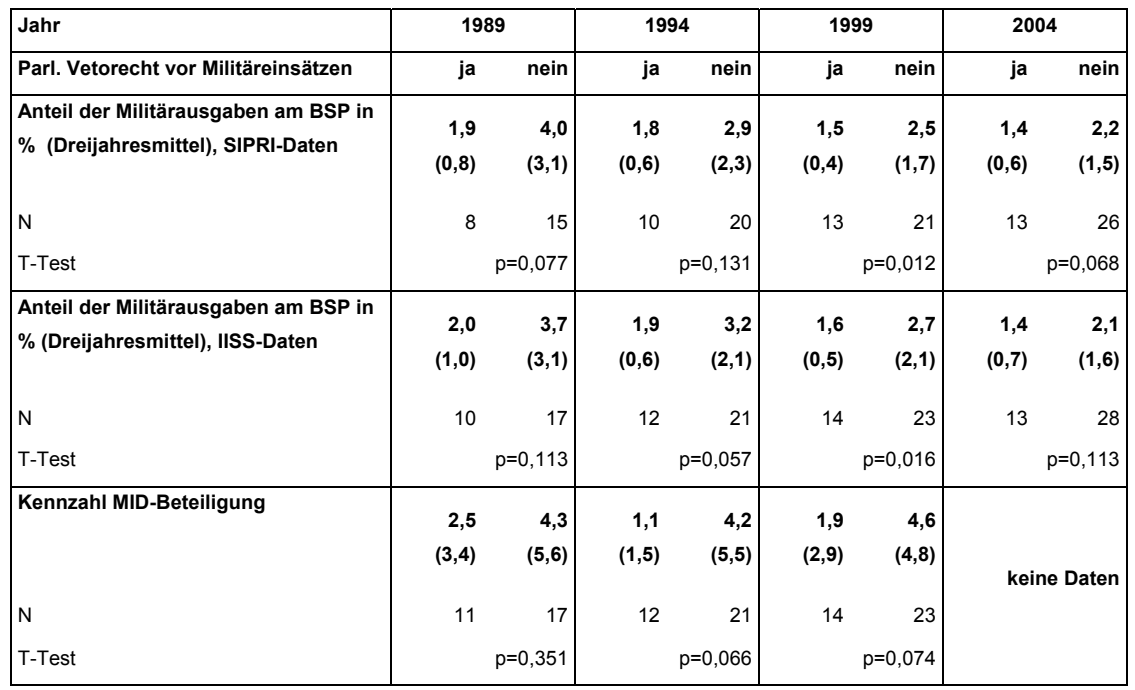

28 Nach den SIPRI-Zahlen sind die Unterschiede zwischen den beiden Staatengruppen in allen Jahren außer 1991-1994 zumindest auf dem 10\%-Niveau statistisch signifikant, nach den Zahlen des IISS in den Jahren 1993-1995 und 1997-2000.

29 In den Jahren 1993-1995 und 1998-1999 ist $p<0,1$.

30 Signifikanzen wurden in jenen Jahren unter der Annahme ungleicher Varianzen berechnet, für die Levenes Test statistisch signifikante Unterschiede $(\mathrm{p}<0,05)$ in den Varianzen anzeigt. Das betrifft für die Variable Militärausgaben (SIPRI- und IISS-Daten) die Jahre 1999-2000. 


\subsection{Demokratietyp-Hypothesen}

Auch hinsichtlich des politischen Systems unterscheiden sich Staaten mit und ohne Vetokompetenz erheblich (Tabelle 5). Zwar spiegelt sich die generelle Unterscheidung zwischen parlamentarischen und präsidentiellen Systemen nicht in den Daten wider. So liegt der Anteil parlamentarischer Systeme sowohl bei den Staaten mit als auch bei jenen ohne Parlamentsveto über die Jahre hinweg bei etwas über 75 Prozent, mit kleineren aber unsystematischen Abweichungen. ${ }^{31}$ Die Commonwealth-Mitglieder ballen sich dagegen geradezu in der Gruppe der Staaten ohne parlamentarisches Vetorecht. Hier ist der Anteil der Commonwealth-Staaten durchweg um ein Vielfaches höher als in der Gruppe der Länder mit Parlamentsveto. Obwohl statistische Signifikanztests hier durch die vergleichsweise geringen Fallzahlen in einzelnen Kategorien erschwert werden, ist dieses Muster immerhin noch in mehr als der Hälfte der untersuchten Jahre statistisch signifikant. ${ }^{32}$ Erkennbar ist der Zusammenhang vor allem daran, dass nur in einem einzigen der elf Commonwealth-Staaten das Parlament durchweg ein Vetorecht über die Entsendung des Militärs genießt, nämlich in PapuaNeuguinea. ${ }^{33}$

\section{Tabelle 5: Verteilung der Demokratietypen; zweiseitige Signifikanztests}

\begin{tabular}{|l|rr|rr|rr|rr|}
\hline Jahr & \multicolumn{2}{|c|}{1989} & \multicolumn{2}{|c|}{$\mathbf{1 9 9 4}$} & \multicolumn{2}{|c|}{$\mathbf{1 9 9 9}$} & \multicolumn{2}{|c|}{$\mathbf{2 0 0 4}$} \\
\hline Parl. Vetorecht vor Militäreinsätzen & ja & nein & ja & nein & ja & nein & ja & nein \\
\hline N parl. Systeme/gesamt & $8 / 11$ & $14 / 17$ & $9 / 12$ & $16 / 21$ & $11 / 14$ & $18 / 23$ & $10 / 13$ & $23 / 28$ \\
Anteil parlamentarische Systeme & $\mathbf{0 , 7 3}$ & $\mathbf{0 , 8 2}$ & $\mathbf{0 , 7 5}$ & $\mathbf{0 , 7 6}$ & $\mathbf{0 , 7 9}$ & $\mathbf{0 , 7 8}$ & $\mathbf{0 , 7 7}$ & $\mathbf{0 , 8 2}$ \\
Exakter Test nach Fisher & & $p=0,653$ & & $p=1,000$ & & $p=1,000$ & $p=0,692$ \\
\hline N Commonwealth/gesamt & $1 / 11$ & $7 / 17$ & $1 / 12$ & $7 / 21$ & $1 / 14$ & $10 / 23$ & $2 / 13$ & $9 / 28$ \\
Anteil Commonwealth-Staaten & $\mathbf{0 , 0 9}$ & $\mathbf{0 , 4 1}$ & $\mathbf{0 , 0 8}$ & $\mathbf{0 , 3 3}$ & $\mathbf{0 , 0 7}$ & $\mathbf{0 , 4 3}$ & $\mathbf{0 , 1 5}$ & $\mathbf{0 , 3 2}$ \\
Exakter Test nach Fisher & & $\mathrm{p}=0,099$ & & $\mathrm{p}=0,206$ & & $\mathrm{p}=0,027$ & & $\mathrm{p}=0,451$ \\
\hline
\end{tabular}

\subsection{Gesamteinschätzung}

Auf bivariater Ebene bewähren sich also drei Hypothesen. Wir erkennen einen Zusammenhang zwischen dem Politikversagen in früheren Konflikten, ablesbar an der Zahl der Gefallenen in verlorenen Kriegen, und der Existenz eines parlamentarischen Vetos über Militäreinsätze; zwischen der Bedrohung, der ein Staat ausgesetzt ist, und den Vetokompetenzen des Parlaments sowie zwischen der britischen Verfassungs-

31 Dies gilt unabhängig davon, ob semi-präsidentielle Systeme, wie in Tabelle 5, als parlamentarische oder aber als präsidentielle System klassifiziert werden.

$32 \mathrm{p}<0,1$ gilt für die Jahre 1989-1990 und 1997-2003.

332004 kommt Zypern hinzu, in dem das Parlament Ende 2003 ein Vetorecht über Militäreinsätze eingeräumt bekam. 
tradition und den untersuchten Parlamentskompetenzen. Für die Bedeutung der Demokratisierung eines Staates und für die Relevanz der Unterscheidung zwischen parlamentarischen und präsidentiellen Systemen konnten wir hingegen keine Belege finden. Um wenigstens abschätzen zu können, ob die untersuchten Variablen auch bei gleichzeitiger Analyse einen Einfluss auf die Existenz eines parlamentarischen Vetorechts haben, führen wir abschließend eine multiple Regression mit den eingangs genannten Einschränkungen durch. Das heißt: Wir beschränken uns auf die Einbeziehung von drei unabhängigen Variablen und die Untersuchung jener Jahre, in denen die Zahl der Länder mit parlamentarischem Vetorecht über 12 liegt. ${ }^{34}$ Dies sind die Jahre 1995-2004 außer 2001. In einer solchen Analyse mit grenzwertig niedriger Fallzahl sind vor allem »Typ II«-Fehler nicht auszuschließen (Vittinghoff/Culloch 2007: 717; McCall Smith 2000: 170) - unter Umständen werden also Hypothesen abgelehnt, obwohl sie in der Grundgesamtheit zutreffen würden. Umgekehrt gilt aber: Wenn Zusammenhänge in der Analyse als statistisch signifikant erkannt werden, kann davon ausgegangen werden, dass sie sich auch in einem größeren Sample bzw. in der Grundgesamtheit nachweisen ließen.

34 Dabei ist zu berücksichtigen, dass die Fallzahlen durch fehlende Werte bei einigen unabhängigen Variablen weiter eingeschränkt werden. SIPRI-Daten liegen für maximal 14 Länder mit parlamentarischem Vetorecht vor (2000, 2002), Daten über die MID-Beteiligung nur für die Jahre bis 2001. 
Tabelle 6: Ergebnisse der logistischen Regression mit der abhängigen Variable "parlamentarisches Vetorecht vor Entsendung des Militärs" $(1=$ vorhanden). Angegeben sind die Chancenverhältnisse (odds ratios), Standardfehler in Klammern

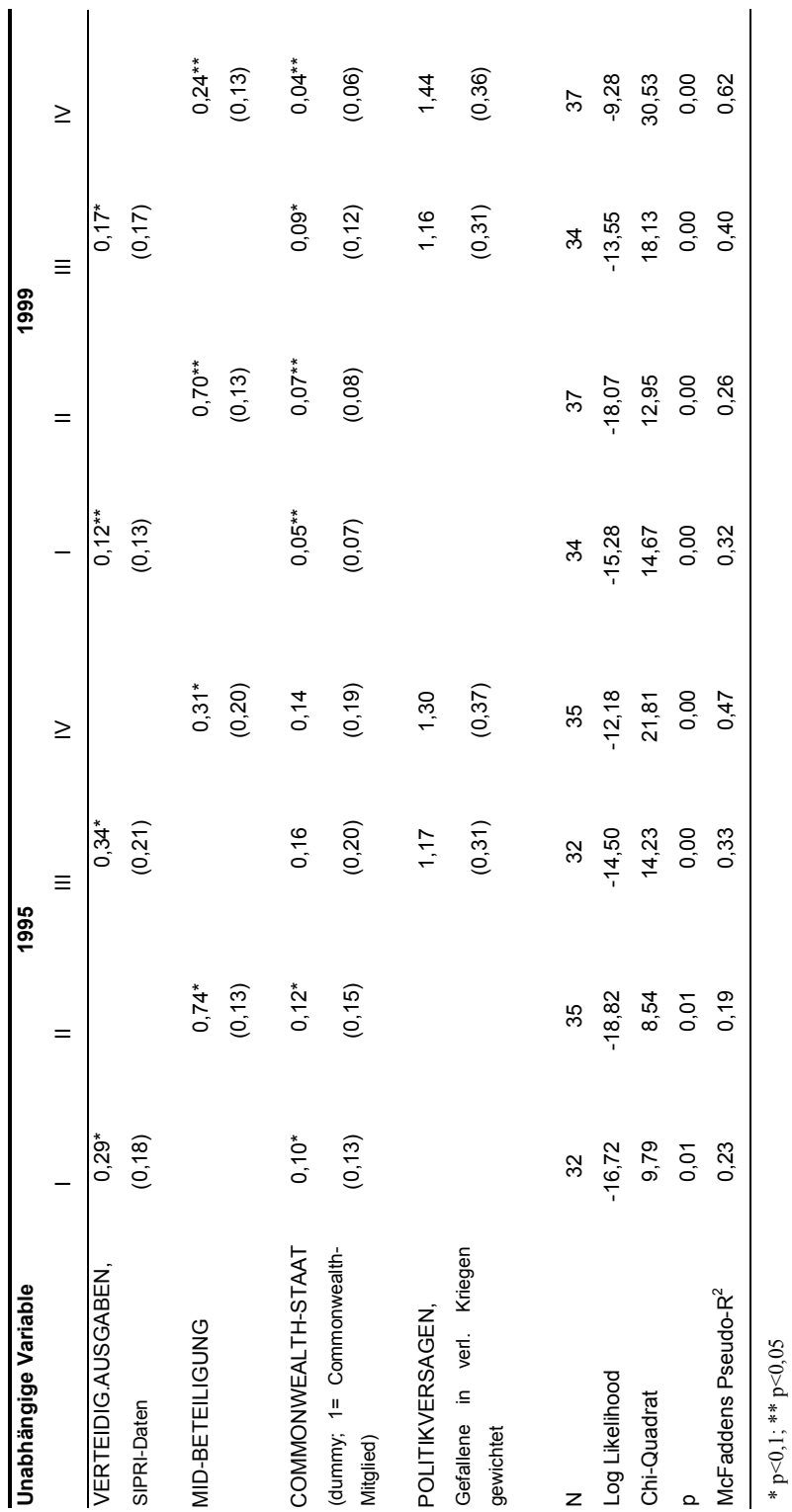


Die Ergebnisse dieser multiplen Regression sind in Tabelle 6 dargestellt. Wir beziehen zunächst die beiden Variablen ein, die in der bivariaten Analyse als die wichtigsten identifiziert wurden, Bedrohung und Zugehörigkeit zum Commonwealth. Bedrohung bestimmen wir dabei jeweils durch einen unserer beiden Indikatoren (Modelle I und II). Die Zahlen zeigen, dass in einer multiplen Regression selbst bei unseren niedrigen Fallzahlen die Zusammenhänge substantiell und statistisch signifikant sind. Dies gilt unabhängig davon, ob die Verteidigungsausgaben oder die Beteiligung an MIDs als Bedrohungsindikator benutzt werden. ${ }^{35}$ Die Hinzunahme der Variable Politikversagen (Modelle III und IV) führt zwar zu einer erkennbaren Verbesserung der Erklärungskraft der Modelle insgesamt. Allerdings bleibt der Koeffizient für die zusätzliche Variable unterhalb der Schwelle statistischer Signifikanz. ${ }^{36}$ Dies liegt nicht zuletzt an einer Korrelation zwischen der Mitgliedschaft im Commonwealth und niedrigen Werten für die Variable Politikversagen: Mitglieder des Commonwealth haben seltener als andere Staaten verlustreiche Kriege verloren. Aufgrund der besonderen Probleme der multiplen logistischen Regression bei solch niedrigen Fallzahlen kann dies aber nicht letztgültig als Scheitern der Lessons-learnt-Hypothese interpretiert werden.

\section{Schlussfolgerungen}

Ob eine Regierung vor dem Einsatz des Militärs die Zustimmung des Parlaments einholen muss oder ob sie eine solche Entscheidung im Alleingang treffen kann, kann das Konfliktverhalten von Demokratien erheblich beeinflussen. Das gilt insbesondere dann, wenn, wie im Falle des Irakkrieges 2003, die öffentliche Meinung mehrheitlich einen Militäreinsatz ablehnt (Dieterich et al. 2009). Die Ursachen für die auffälligen Unterschiede zwischen »pazifistischen« und »bellizistischen« Demokratien liegen nicht nur in der politischen Kultur (Müller 2004), sondern darüber hinaus in den jeweiligen Institutionen, die die Handlungsfreiheiten der Exekutive festlegen und die Übersetzung pazifistischer Präferenzen in der Bürgerschaft in Regierungspolitik erleichtern bzw. erschweren (Owen 1994).

Vor diesem Hintergrund kann das gestiegene Interesse der Konfliktforschung an den Unterschieden zwischen Demokratien kaum überraschen. Ziel dieses Beitrags ist es, diese Forschung einen Schritt weiter zu führen und danach zu fragen, wie es überhaupt zu derartigen Unterschieden zwischen Demokratien kommen kann. Er fokussiert auf die An- bzw. Abwesenheit eines parlamentarischen Vetorechts über Militäreinsätze und konzentriert sich bei der Ursachensuche darauf, grundlegende und verallgemeinerbare Muster zu identifizieren.

35 Dies gilt für Modell I in allen untersuchten Jahren außer 2004 und für Modell II in allen untersuchten Jahren außer 1996 und 1997.

36 Dies gilt in allen untersuchten Jahren. Außerdem erreicht der Koeffizient für die Variable Commonwealth das 10\%-Signifikanzniveau nur noch in den Jahren 1997-2000 (Modell III) bzw. 1998-2000 (Modell IV). 
Trotz der methodischen Schwierigkeiten, die mit der begrenzten Fallzahl und einem hohen Maß an Autokorrelation über Zeit zusammenhängen, können wir hierbei zwei Befunde mit Verlässlichkeit festhalten: Zum einen erhöht sich mit dem Ausmaß externer Bedrohung die Wahrscheinlichkeit, dass Entscheidungen über Militäreinsätze ein alleiniges Privileg der Exekutive sind und dem Parlament kein Vetorecht zugestanden wird. Dieser Befund zeigt sich unabhängig davon, ob Bedrohung anhand der Zahl von Konflikten gemessen wird, in die ein Staat verwickelt war, oder ob anhand der Verteidigungsausgaben der Bedrohungswahrnehmung eine bedeutsamere Rolle beigemessen wird. Zum zweiten setzt sich in den Commonwealth-Staaten jene Tradition der britischen Verfassung fort, nach der die Außen- und Sicherheitspolitik als Privileg der Exekutive zu betrachten ist. Es gibt außerdem Indizien dafür, dass auch vergangene Kriegserfahrungen eine Rolle für die Gestaltung der parlamentarischen Mitspracheregeln spielen. Staaten, die in der Vergangenheit verlustreiche Kriege verloren haben, tendieren zu einem parlamentarischen Veto über Militäreinsätze.

Alles in allem ergibt sich so ein komplexes Muster, in dem die sicherheitspolitische Rolle des Parlaments weniger von generellen Aspekten des politischen Systems (wie der Unterscheidung zwischen parlamentarischen und präsidentiellen Demokratien), sondern vielmehr von spezifischen kulturellen Gegebenheiten und Erfahrungen gemeinsam mit der Bedrohung, der sich ein Staat ausgesetzt sieht, beeinflusst wird. So erweist sich zunächst die Verfassungs- und die Konfliktgeschichte eines Landes als wichtig. Ein parlamentarisches Veto über Militäreinsätze ist fast nur in Ländern zu finden, die keine direkte Verbindung zur britischen Verfassungstradition haben. Erfahrungen mit verlorenen Kriegen, die zahlreiche eigene Opfer gefordert haben, tragen hingegen tendenziell zur Einrichtung eines parlamentarischen Vetos bei. Evidenz für dieses Muster findet sich zum Beispiel in Staaten wie Deutschland und Japan, wo die Erfahrungen des Zweiten Weltkriegs zu großen Vorbehalten gegen den Einsatz militärischer Gewalt durch die Exekutive geführt haben, die sich verfassungsrechtlich in eine starke Rolle des Parlaments übersetzt haben.

Hinzu kommt die Wirksamkeit des sicherheitspolitischen Umfelds einer Demokratie. Der Zusammenhang zwischen Bedrohung und Stellung des Parlaments bei der Entscheidung über den Einsatz des Militärs lässt sich gut am Beispiel Israels illustrieren: Israel befindet sich in einer äußerst prekären Sicherheitslage und das Militär steht verfassungsrechtlich selbstverständlich unter der Entscheidungsgewalt der Exekutive, während das Parlament auch in der Praxis nur geringe Kontrollfunktionen wahrnimmt (Maoz 2006: 527-530). Unsere Befunde bestätigen in dieser Hinsicht die second image reversed-These, wonach der innere Aufbau von Staaten von ihrer Position im internationalen System beeinflusst ist. Da die Entscheidungsverfahren in Demokratien wiederum nachweislich ihr Konfliktverhalten beeinflussen, wie die Forschung zum Demokratischen Frieden gezeigt hat, können sich hier wechselseitig verstärkende Zusammenhänge ergeben: In einer friedlichen Umwelt neigen Demokratien eher dazu, Parlamenten ein Vetorecht bei Militäreinsätzen zu geben, was wiederum vor allem in Konstellationen, in denen die Regierung bellizistischer ist als die Bürgerschaft, eine friedliche Außenpolitik wahrscheinlicher macht. Umgekehrt verfügen Regierungen in einer bedrohlichen Umwelt häufiger über große Entschei- 
dungsspielräume beim Einsatz des Militärs, was wiederum die Verwicklung in militarisierte Konflikte wahrscheinlicher macht.

Die hier vorgestellten Daten können nicht nur verwendet werden, um generalisierbare Muster zu finden, sie können auch die Grundlage für gezielte Einzelfallstudien bilden. Naheliegende Beispiele für solche Fälle sind jene Staaten, in denen sich die parlamentarischen Vetorechte im Laufe des Untersuchungszeitraums verändert haben. Einzelfallstudien könnten hier helfen, die Ursachen für diese Veränderungen im Detail nachzuzeichnen. Während eine umfassende Analyse dieser Fälle den Rahmen dieses Beitrags bei weitem sprengen würde, legt ein erster Blick auf diese Fälle die Vermutung nahe, dass hier auch Variablen von Bedeutung gewesen sein können, über deren grundsätzliche Wirkung aufgrund der niedrigen Fallzahlen in der vorliegenden Untersuchung keine Aussagen gemacht werden können. So sind vier der fünf Länder, in denen eine solche Veränderung stattfand, mittel- und osteuropäische Staaten, die zunächst nach ihrer Transition zur Demokratie ein Parlamentsveto über Militäreinsätze institutionalisierten, wie von der Demokratisierungs-Hypothese erwartet. Alle vier Staaten, Ungarn, Tschechien, die Slowakei und Bulgarien, schränkten die Parlamentsrechte später aber wieder ein. Bei allen vier Staaten scheint dabei der Beitritt zur NATO eine wichtige Rolle gespielt zu haben. Kritik aus der NATO, die verfassungsrechtlichen Regeln behinderten eine Teilnahme an gemeinsamen NATO-Operationen, ging bspw. der ungarischen Reform der Parlamentsrechte voraus (Dunay 2004; 2005). Die Neuinterpretation der Parlamentsrechte durch das Verfassungsgericht in Bulgarien erfolgte, um Bulgariens Beteiligung an NATO-Operationen zu erleichtern (Wagner et al. 2010: 35-36), ebenso wie die Neufassung der tschechischen und der slowakischen Regeln zur Parlamentsbeteiligung (Simon 2004: 79-93, 219).

Mit Hilfe von Einzelfallanalysen ließen sich genauere Aussagen über die Bedeutung solcher spezifischen und nur für eine kleinere Zahl von Fällen existierenden Variablenkombinationen machen. Über alle Fälle verallgemeinerbar hingegen sind die Zusammenhänge, denen sich dieser Beitrag schwerpunktmäßig gewidmet hat.

\section{Literatur}

Auerswald, David P. 1999: Inward Bound: Domestic Institutions and Military Conflicts, in: International Organization 53: 2, 469-504.

Auerswald, David P. 2001: Disarmed Democracies: Domestic Institutions and the Use of Force, Ann Arbor, MI.

Beck, Thorsten/Clarke, George/Groff, Alberto/Keefer, Philip/Walsh, Patrick 2001: New Tools in Comparative Political Economy: The Database of Political Institutions, in: The World Bank Economic Review 15: 1, 165-176.

Born, Hans/Urscheler, Marlene 2004: Parliamentary Accountability of Multinational Peace Support Operations: A Comparative Perspective, in: Born, Hans/Hänggi, Heiner (Hrsg.): The »Double Democratic Deficit«: Parliamentary Accountability and the Use of Force under International Auspices, Aldershot, 53-72.

Born, Hans/Hänggi, Heiner 2005: Governing the Use of Force under International Auspices: Deficits in Parliamentary Accountability, in: SIPRI Yearbook 2005: Armaments, Disarmaments and International Security, Oxford, 199-222. 
Bremer, Stuart 1992: Dangerous Dyads: Conditions Affecting the Likelihood of Interstate War, 1816-1965, in: Journal of Conflict Resolution 36: 2, 309-341.

Brissa, Enrico 2005: Die italienischen Streitkräfte und ihr internationales Engagement. Eine rechtliche, politikwissenschaftliche und historische Untersuchung, Frankfurt a. M.

Bueno de Mesquita, Bruce/Morrow, James/Siverson, Randolph/Smith, Alastair 1999: An Institutional Explanation of the Democratic Peace, in: American Political Science Review 93: 4, 791-807.

Chan, Steve 1984: Mirror, Mirror on the Wall... Are the Freer Countries More Pacific?, in: Journal of Conflict Resolution 28: 4, 617-648.

Chiozza, Giacomo/Goemans, Hein 2004: International Conflict and the Tenure of Leaders: Is War Still Ex Post Inefficient?, in: American Journal of Political Science 48: 3, 604-619.

Collier, Ellen C. 1994: Statutory Constraints: The War Powers Resolution, in: Stern, Gary M./ Halperin, Morton H. (Hrsg.): The US Constitution and the Power to Go to War: Historical and Current Perspectives, Westport, CT, 55-82.

Dembinski, Matthias 2007: Vom Diplomatischen Club zum außenpolitischen Regieren in Netzwerken? Der Einfluss gesellschaftlicher Akteure auf die Gemeinsame Außen- und Sicherheitspolitik am Beispiel der EU-Konfliktprävention, in: Jopp, Matthias/Schlotter, Peter (Hrsg.): Die Gemeinsame Außen- und Sicherheitspolitik (GASP) der Europäischen Union - Intergouvernementales Netzwerk oder kollektiver Akteur?, Baden-Baden, 91-119.

de Tocqueville, Alexis 1990 [1835]: Democracy in America, Band I, New York, NY.

Dieterich, Sandra/Hummel, Hartwig/Marschall, Stefan 2009: »Kriegsspielverderber «? Europäische Parlamente und der Irakkrieg 2003, in: Zeitschrift für Internationale Beziehungen 14: 1, 5-38.

Doyle, Michael W. 1983: Kant, Liberal Legacies, and Foreign Affairs, in: Philosophy and Public Affairs 12: 3/4, 205-235/323-353.

Dunay, Pal 2004: Hungary, in: Giessmann, Hans J. (Hrsg.): Security Handbook 2004. BadenBaden, 147-179.

Dunay, Pal 2005: The Half-hearted Transformation of the Hungarian Military, in: European Security 14: 1, 17-32.

Eichenberg, Richard/Lebo, Matthew/Stoll, Richard 2006: War President: The Approval Ratings of George W. Bush, in: Journal of Conflict Resolution 50: 6, 783-808.

Elman, Miriam Fendius 2000: Unpacking Democracy: Presidentialism, Parliamentarianism, and Theories of Democratic Peace, in: Security Studies 9: 4, 91-126.

Farber, Henry/Gowa, Joanne 1995: Polities and Peace, in: International Security 20: 2, 123-146.

Ganguly, Sumit 2001: Conflict Unending: India-Pakistan Tensions Since 1947, New York, NY.

Gartzke, Erik 2007: The Capitalist Peace, in: American Journal of Political Science 51: 1, 166-191.

Gaubatz, Kurt 1996: Democratic States and Commitment in International Relations, in: International Organization 50: 1, 109-139.

Geis, Anna/Wagner, Wolfgang 2010: How Far is It from Königsberg to Kandahar? Democratic Peace and Democratic Violence in International Relations, in: Review of International Studies, i.E.

Gochman, Charles/Maoz, Zeev 1984: Militarized Interstate Disputes 1816-1976, in: Journal of Conflict Resolution 28: 4, 585-616.

Gourevitch, Peter 1978: The Second Image Reversed: The International Sources of Domestic Politics, in: International Organization 32: 4, 881-912.

Hänggi, Heiner 2004: The Use of Force under International Auspices: Parliamentary Accountbility and »Democratic Deficits«, in: Born, Hans/Hänggi, Heiner (Hrsg.): The »Double Democratic Deficit«: Parliamentary Accountability and the Use of Force under International Auspices, Aldershot, 3-16.

Hall, Peter A. 1993: Policy Paradigms, Social Learning, and the State: The Case of Economic Policymaking in Britain, in: Comparative Politics 25: 3, 275-296. 
Harbom, Lotta/Wallensteen, Peter 2007: Armed Conflicts 1989-2006, in: Journal of Peace Research 44: 5, 623-634.

Harfst, Philipp/Schnapp, Kai-Uwe 2003: Instrumente parlamentarischer Kontrolle der Exekutive in westlichen Demokratien (Discussion Paper SP IV 2003-201), Berlin.

Hasenclever, Andreas 2001: Die Macht der Moral in der internationalen Politik, Frankfurt a. M.

Kant, Immanuel 1984 [1795]: Zum ewigen Frieden, Stuttgart.

King, Anthony 1976: Modes of Executive-Legislative Relations: Great Britain, France, and West Germany, in: Legislative Studies Quarterly 1: 1, 11-36.

Krebs, Ronald R. 2009: In the Shadow of War: The Effects of Conflict on Liberal Democracy, in: International Organization 63: 1, 177-210.

Krebs, Ronald R. 2010: International Conflict and the Constitutional Balance: Executive Authority after War, in: Kier, Elizabeth/Krebs, Ronald R. (Hrsg.): In War's Wake: International Conflict and the Fate of Liberal Democracy, Cambridge, 187-210.

Layne, Christopher 1994: Kant or Cant: The Myth of the Democratic Peace, in: International Security 19: 2, 5-49.

Linz, Juan 1990: The Virtues of Parliamentarism, in: Journal of Democracy 1: 4, 84-91.

Lipson, Charles 2003: Reliable Partners: How Democracies Have Made a Separate Peace, Princeton, NJ.

Locke, John 1960 [1690]: Two Treatises of Government, hrsg. von Peter Laslett, Cambridge.

Lord, Christopher 2008: Is There a Role for Parliamentary Participation in European Security Co-ordination?, in: Peters, Dirk/Wagner, Wolfgang/Deitelhoff, Nicole (Hrsg.): The Parliamentary Control of European Security Policy, Oslo, 51-76.

Mansfield, Edward D./Pevehouse, Jon C. 2006: Democratization and International Organizations, in: International Organization 60: 1, 137-167.

Mansfield, Edward D./Pevehouse, Jon C. 2008: Democratization and the Varieties of International Organizations, in: Journal of Conflict Resolution 52: 2, 269-294.

Mansfield, Edward/Snyder, Jack 1995: Democratization and the Danger of War, in: International Security 20: 1, 5-38.

Mansfield, Edward/Snyder, Jack 2005: Electing to Fight: Why Emerging Democracies Go to War, Cambridge, MA.

Maoz, Zeev 2006: Defending the Holy Land: A Critical Analysis of Israel's Security and Foreign Policy, Ann Arbor, MI.

Martin, Lisa 2000: Democratic Commitments: Legislatures and International Cooperation, Princeton, NJ.

McCall Smith, James 2000: The Politics of Dispute Settlement Design: Explaining Legalism in Regional Trade Pacts, in: International Organization 54: 1, 137-180.

Midlarsky, Manus 1995: Environmental Influences on Democracy: Aridity, Warfare and a Reversal in the Causal Arrow, in: Journal of Conflict Resolution 39: 2, 224-262.

Moravcsik, Andrew 2000: The Origins of Human Rights Regimes: Democratic Delegation in Postwar Europe, in: International Organization 54: 2, 217-252.

Morgan, T. Clifton/Campbell, Sally Howard 1991: Domestic Structure, Decisional Constraints, and War: So Why Kant Democracies Fight?, in: Journal of Conflict Resolution 35: 2, 187-211.

Mueller, John E. 1973: War, Presidents, and Public Opinion, New York, NY.

Müller, Harald 2004: The Antinomy of Democratic Peace, in: International Politics 41: 4, 494-520.

Owen, John 1994: How Liberalism Produces Democratic Peace, in: International Security 19: 2, 87-125.

Peduzzi, Peter/Concato, John/Kemper, Elizabeth/Holford, Theodore R./Seinstein, Alvan R. 1996: A Simulation Study of the Number of Events per Variable in Logistic Regression Analysis, in: Journal of Clinical Epidemiology 49: 12, 1373-1379. 
Peters, Dirk/Wagner, Wolfgang 2011: Between Military Efficiency and Democratic Legitimacy: Mapping Parliamentary War Powers in Contemporary Democracies, 1989-2004, in: Parliamentary Affairs 64, i.E.

Rasler, Karen/Thompson, William 2005: Puzzles of the Democratic Peace: Theory, Geopolitics, and the Transformation of World Politics, Basingstoke.

Reiter, Dan/Stam, Allen 2002: Democracies at War, Princeton, NJ.

Risse-Kappen, Thomas 1995: Democratic Peace - Warlike Democracies? A Social Constructivist Interpretation of the Democratic Peace, in: European Journal of International Relations 1: 4, 491-517.

Rosato, Sebastian 2003: The Flawed Logic of Democratic Peace Theory, in: American Political Science Review 97: 4, 585-602.

Rothermund, Dietmar 2002: Krisenherd Kaschmir. Der Konflikt der Atommächte Indien und Pakistan, München.

Rummel, Rudolph 1995: Democracies are Less Warlike Than Other Regimes, in: European Journal of International Relations 1: 4, 457-479.

Russett, Bruce 1993: Grasping the Democratic Peace: Principles for a Post-Cold War World, Princeton, NJ.

Russett, Bruce/Oneal, John 2001: Triangulating Peace: Democracy, Interdependence and International Organization, New York, NY.

Schörnig, Niklas 2007: Die Vision des wohlfeilen Krieges. Hightech-Antworten zur Umgehung der Opfersensibilitätsfalle, in: Geis, Anna/Müller, Harald/Wagner, Wolfgang (Hrsg.): Schattenseiten des Demokratischen Friedens, Frankfurt a. M., 93-122.

Schwarzmeier, Manfred 2001: Parlamentarische Mitsteuerung. Strukturen und Prozesse informalen Einflusses im Deutschen Bundestag, Wiesbaden.

Simon, Jeffrey 2004: NATO and the Czech and Slovak Republics: A Comparative Study in CivilMilitary Relations, Lanham, MD.

Siverson, Randolph M. 1995: Democracies and War Participation: In Defense of the Institutional Constraints Argument, in: European Journal of International Relations 1: 4, 481-489.

Steffani, Winfried 1979: Parlamentarische und präsidentielle Demokratie, Opladen.

Strøm, Kaare 2000: Delegation and Accountability in Parliamentary Democracies, in: European Journal of Political Research 37: 3, 261-289.

Thompson, William 1996: Democracy and Peace: Putting the Cart before the Horse?, in: International Organization 50: 1, 141-174.

Vittinghoff, Eric/McCulloch, Charles E. 2007: Relaxing the Rule of Ten Events per Variable in Logistic and Cox Regression, in: American Journal of Epidemiology 165: 6, 710-718.

Wagner, Wolfgang/Peters, Dirk/Glahn, Cosima 2010: Parliamentary War Powers Around the World, 1989-2004: A New Dataset (Geneva Center for the Democratic Control of Armed Forces (DCAF) Occasional Paper 22), Genf.

Walsh, James Igoe 2007: How and Why Britain Might Join the Single Currency: The Role of Policy Failure, in: Review of International Political Economy 14: 5, 868-892.

Waltz, Kenneth N. 1959: Man, the State, and War: A Theoretical Analysis, New York, NY.

Watts, Stephen 2008: Air War and Restraint: The Role of Public Opinion and Democracy, in: Evangelista, Matthew/Müller, Harald/Schörnig, Niklas (Hrsg.): Democracy and Security, London, 53-71.

Weart, Spencer 1998: Never at War: Why Democracies Will Not Fight One Another, New Haven, $\mathrm{CO}$.

White, Nigel 2003: The United Kingdom: Increasing Commitment Requires Greater Parliamentary Involvement, in: Ku, Charlotte/Jacobson, Harold (Hrsg.): Democratic Accountability and the Use of Force in International Law, Cambridge, 300-322. 
Appendix: Parlamentarische Vetorechte bei Militäreinsätzen

\begin{tabular}{|c|c|c|}
\hline Land & Zeitraum & Vetorecht bei der Entsendung von Streitkräften \\
\hline Australien & $1989-2004$ & Nicht vorhanden \\
\hline Belgien & $1989-2004$ & Nicht vorhanden \\
\hline Bolivien & $1989-2002$ & Vorhanden \\
\hline Botswana & $1997-2004$ & Nicht vorhanden \\
\hline Bulgarien & $2001-2004$ & $\begin{array}{l}\text { Vorhanden (2001-2002) } \\
\text { Nicht vorhanden }(2003-2004)\end{array}$ \\
\hline Chile & $2000-2004$ & $\begin{array}{l}\text { Vorhanden }(2000-2003) \\
\text { Unbestimmbar (2004) }\end{array}$ \\
\hline Dänemark & $1989-2004$ & Vorhanden \\
\hline Deutschland & $1989-2004$ & $\begin{array}{l}\text { Unbestimmbar (1989-1994) } \\
\text { Vorhanden (1995-2004) }\end{array}$ \\
\hline Ecuador & $\begin{array}{l}1989-1996, \\
1998-1999\end{array}$ & Nicht vorhanden \\
\hline Finnland & $1989-2004$ & Vorhanden \\
\hline Frankreich & $1989-2004$ & Nicht vorhanden \\
\hline Griechenland & $1989-2004$ & Nicht vorhanden \\
\hline Indien & $1995-2004$ & Nicht vorhanden \\
\hline Irland & $1989-2004$ & Vorhanden \\
\hline Israel & $1989-2004$ & Nicht vorhanden \\
\hline Italien & $1989-2004$ & Unbestimmbar \\
\hline Jamaika & $1989-2004$ & Nicht vorhanden \\
\hline Japan & $1989-2004$ & Vorhanden \\
\hline Kanada & $1989-2004$ & Nicht vorhanden \\
\hline Kolumbien & 1991-1994 & Nicht vorhanden \\
\hline Litauen & $1991-2004$ & $\begin{array}{l}\text { Unbestimmbar (1991-1992) } \\
\text { Vorhanden (1993-2004) }\end{array}$ \\
\hline Madagaskar & $1992-1996$ & Nicht vorhanden \\
\hline Mazedonien & $2002-2004$ & Vorhanden \\
\hline
\end{tabular}




\begin{tabular}{|c|c|c|}
\hline Land & Zeitraum & Vetorecht bei der Entsendung von Streitkräften \\
\hline Mongolei & $1992-2004$ & $\begin{array}{l}\text { Unbestimmbar (1992-2001) } \\
\text { Nicht vorhanden }(2002-2004)\end{array}$ \\
\hline Neuseeland & $1989-2004$ & Nicht vorhanden \\
\hline Niederlande & $1989-2004$ & Nicht vorhanden \\
\hline Norwegen & $1989-2004$ & Nicht vorhanden \\
\hline Österreich & $1989-2004$ & Vorhanden \\
\hline $\begin{array}{l}\text { Papua-Neu } \\
\text { Guinea }\end{array}$ & $1989-2004$ & Vorhanden \\
\hline Peru & 2001-2004 & Nicht vorhanden \\
\hline Polen & $1995-2004$ & Nicht vorhanden \\
\hline Portugal & $1989-2004$ & Nicht vorhanden \\
\hline Rumänien & 2004 & Nicht vorhanden \\
\hline Schweden & $1989-2004$ & Vorhanden \\
\hline Schweiz & $1989-2004$ & $\begin{array}{l}\text { Unbestimmbar (1989-2001) } \\
\text { Vorhanden (2002-2004) }\end{array}$ \\
\hline Slowakei & $1998-2004$ & $\begin{array}{l}\text { Vorhanden (1998-2000) } \\
\text { Nicht vorhanden }(2001-2004)\end{array}$ \\
\hline Slowenien & $1991-2004$ & Nicht vorhanden \\
\hline Spanien & 1989-2004 & Nicht vorhanden \\
\hline Südafrika & 1994-2004 & $\begin{array}{l}\text { Unbestimmbar (1994-1996) } \\
\text { Nicht vorhanden (1997-2004) }\end{array}$ \\
\hline Thailand & $1992-2004$ & Nicht vorhanden \\
\hline $\begin{array}{l}\text { Trinidad und } \\
\text { Tobago }\end{array}$ & 1989-2004 & Nicht vorhanden \\
\hline $\begin{array}{l}\text { Tschechische } \\
\text { Republik }\end{array}$ & 1993-2004 & $\begin{array}{l}\text { Vorhanden }(1993-2000) \\
\text { Nicht vorhanden }(2001-2004)\end{array}$ \\
\hline Türkei & 1989-1992 & Vorhanden \\
\hline Ungarn & $1990-2004$ & $\begin{array}{l}\text { Vorhanden (1990-2003) } \\
\text { Nicht vorhanden (2004) }\end{array}$ \\
\hline USA & $1989-2004$ & Nicht vorhanden \\
\hline Uruguay & 1989-2004 & Vorhanden \\
\hline Venezuela & 1989-1991 & Vorhanden \\
\hline
\end{tabular}


Aufsätze

\begin{tabular}{|l|c|l|}
\hline Land & Zeitraum & Vetorecht bei der Entsendung von Streitkräften \\
\hline $\begin{array}{l}\text { Vereinigtes } \\
\text { Königreich }\end{array}$ & $1989-2004$ & Nicht vorhanden \\
\hline Zypern & $1989-2004$ & $\begin{array}{l}\text { Nicht vorhanden (1989-2003) } \\
\text { Vorhanden (2004) }\end{array}$ \\
\hline
\end{tabular}

